\title{
RELAP5 Analysis of OECD/NEA ROSA Project Experiment Simulating a PWR Loss-of-Feedwater Transient with High-Power Natural Circulation
}

\author{
Takeshi Takeda, ${ }^{1}$ Hideaki Asaka, ${ }^{2}$ and Hideo Nakamura ${ }^{1}$ \\ ${ }^{1}$ Nuclear Safety Research Center, Japan Atomic Energy Agency (JAEA), Tokai-mura, Ibaraki-ken 319-1195, Japan \\ ${ }^{2}$ Nuclear Energy System Safety Division, Japan Nuclear Energy Safety Organization (JNES), Minato-ku, Tokyo 105-0001, Japan
}

Correspondence should be addressed to Takeshi Takeda, takeda.takeshi@jaea.go.jp

Received 6 August 2011; Accepted 12 November 2011

Academic Editor: Klaus Umminger

Copyright (C) 2012 Takeshi Takeda et al. This is an open access article distributed under the Creative Commons Attribution License, which permits unrestricted use, distribution, and reproduction in any medium, provided the original work is properly cited.

\begin{abstract}
A ROSA/LSTF experiment was conducted for OECD/NEA ROSA Project simulating a PWR loss-of-feedwater (LOFW) transient with specific assumptions of failure of scram that may cause natural circulation with high core power and total failure of high pressure injection system. Auxiliary feedwater (AFW) was provided to well observe the long-term high-power natural circulation. The core power curve was obtained from a RELAP5 code analysis of PWR LOFW transient without scram. The primary and steam generator (SG) secondary-side pressures were maintained, respectively, at around 16 and $8 \mathrm{MPa}$ by cycle opening of pressurizer (PZR) power-operated relief valve and SG relief valves for a long time. Large-amplitude level oscillation occurred in SG U-tubes for a long time in a form of slow fill and dump while the two-phase natural circulation flow rate gradually decreased with some oscillation. RELAP5 post-test analyses were performed to well understand the observed phenomena by employing a fine-mesh multiple parallel flow channel representation of SG U-tubes with a Wallis counter-current flow limiting correlation at the inlet of U-tubes. The code, however, has remaining problems in proper predictions of the oscillative primary loop flow rate and SG U-tube liquid level as well as PZR liquid level.
\end{abstract}

\section{Introduction}

High reliability of control rods results in relatively low risk for anticipated transient without scram (ATWS) of pressurized water reactor (PWR). Failure of scram during loss-of-feedwater (LOFW) transient, however, should lead to relatively high core power for a long time and significant thermal-hydraulic responses which may cause degradation in core cooling with gradual loss of primary coolant inventory. Such phenomena include high-power natural circulation with liquid entrainment in hot leg at the inlet of pressurizer (PZR) surge-line, and counter-current flow limiting (CCFL) at the PZR bottom that may hold a large amount of coolant in the PZR, as shown in Figure 1. In the transient following LOFW, power-operated relief valve (PORV) of PZR may continue cycle opening, resulting in loss of primary coolant inventory. The core cooling conditions would then be degraded especially after the natural circulation mode turns into reflux cooling.
A LOFW-induced ATWS experiment was conducted in the LOFT (Loss of Fluid Test) program in the USA and revealed that the primary pressure is kept below about 17.2 MPa by cycle opening of the PZR PORV and safety valve while the primary fluid temperature gradually increases [1]. A LOFW-ATWS experiment in the LOBI (loop-blowdown investigations) facility in Italy showed that cycle opening of the PORV causes depletion of primary coolant inventory resulting in no plant recovery while auxiliary feedwater $(\mathrm{AFW})$ is actuated just after the initiation of core dryout [2]. Experimental data, however, have been scarcely obtained for such a LOFW transient without scram where the thermalhydraulic phenomena change as the primary coolant inventory gradually decreases with time. An experiment on LOFW transient with scram simulating PWR TMLB' scenario [3] was performed with the Large Scale Test Facility (LSTF) [4] at JAEA in 1988. The TMLB' scenario involves prolonged complete loss of AC power, including the off-site power and the on-site emergency diesel generator power, and 
unavailability of turbine-driven AFW. The test on LOFW with scram revealed that two-phase natural circulation continues for a long time until significant drop starts in liquid levels in upflow side of steam generator (SG) U-tubes $[5]$.

OECD/NEA ROSA Project experiment using the LSTF was conducted simulating a PWR LOFW transient with assumptions of high-power natural circulation due to failure of scram and total failure of high pressure injection (HPI) system. The main test objectives are to clarify thermalhydraulic phenomena specific to natural circulation under high core power condition due to failure of scram during the LOFW transient and to obtain the detailed data on the complicated coolant behaviors during natural circulation which are suitable for the validation of best-estimate computer codes (e.g., RELAP5 code). A test condition was similar to TMLB' scenario but with AFW actuation to well observe two-phase natural circulation under high core power for relatively long time. The core power curve for the LSTF experiment was obtained by analysis for PWR LOFW transient without scram using RELAP5/MOD3.2.1.2 code [6] with one-point neutron kinetics model. It was confirmed through SKETCH-INS/TRAC-PF1 code analysis [7] that influences of three-dimensional neutron flux distribution onto core power should be small due to entire core covery by two-phase mixture. Posttest analyses for the LSTF experiment were performed by the RELAP5 code to clarify the details of major phenomena and to validate the code predictability. This paper describes major observations in the LSTF experiment and the RELAP5 post-test analysis results to understand thermal-hydraulic responses during LOFW with high-power natural circulation.

\section{OECD/NEA ROSA Project}

The JAEA started OECD/NEA ROSA Project in 2005 to resolve issues in thermal-hydraulic analyses relevant to light water reactor (LWR) safety by using the LSTF of JAEA [8]. Eighteen organizations from 14 NEA member countries have joined the ROSA Project to date. The OECD/NEA ROSA Project intends to focus on the validation of simulation models and methods for complex phenomena such as multidimensional mixing, stratification, parallel flows, and oscillatory flows with or without influences of noncondensable gas, which may occur during design basis events (DBEs) and beyond-DBE transients.

The experimental program is defined to provide valuable and broadly usable database to achieve the objectives agreed among participants. The ROSA Project consists of twelve LSTF experiments that include the following six types:

(1) temperature stratification and coolant mixing during emergency core cooling system (ECCS) coolant injection,

(2) unstable and destructive phenomena such as water hammer,

(3) natural circulation under high core power conditions,

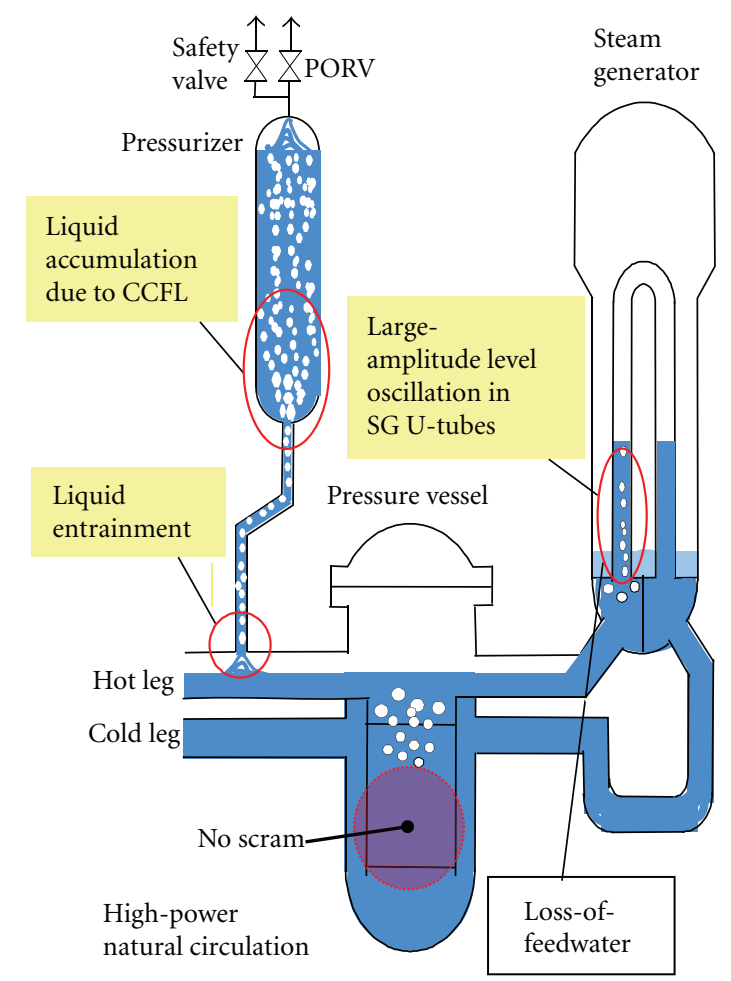

FIGURE 1: Thermal-hydraulic phenomena during LOFW transient without scram.

(4) natural circulation with superheated steam,

(5) primary cooling through SG secondary depressurization,

(6) request-based experiments such as small-break lossof-coolant accident (LOCA) with break at the top or bottom of pressure vessel both being coupled with accident management measures with symptomoriented operator actions and steam condensation on ECCS coolant during large-break LOCA.

This paper concerns the test and analysis results of LOFW transient without scram regarding the third item listed above.

\section{ROSA/LSTF}

The ROSA/LSTF is the world largest integral test facility designed to investigate multi-dimensional thermal-hydraulic responses during PWR transients and accidents. The LSTF simulates a Westinghouse-type four-loop $3423 \mathrm{MWt}$ PWR by a two-loop system model with full-height and 1/48 in volume. The reference PWR is Tsuruga Unit-2 of Japan Atomic Power Company (JAPC). Experiments can be performed under wide range of primary and secondary pressures from reactor nominal operating pressures of $16 \mathrm{MPa}$ and $8 \mathrm{MPa}$, respectively, to atmospheric pressure.

Figure 2 shows the schematic view of the LSTF that is composed of pressure vessel, PZR, and primary loops. An active SG, primary coolant pump, hot and cold legs are included in each loop. Each SG is furnished with 141 full-size 


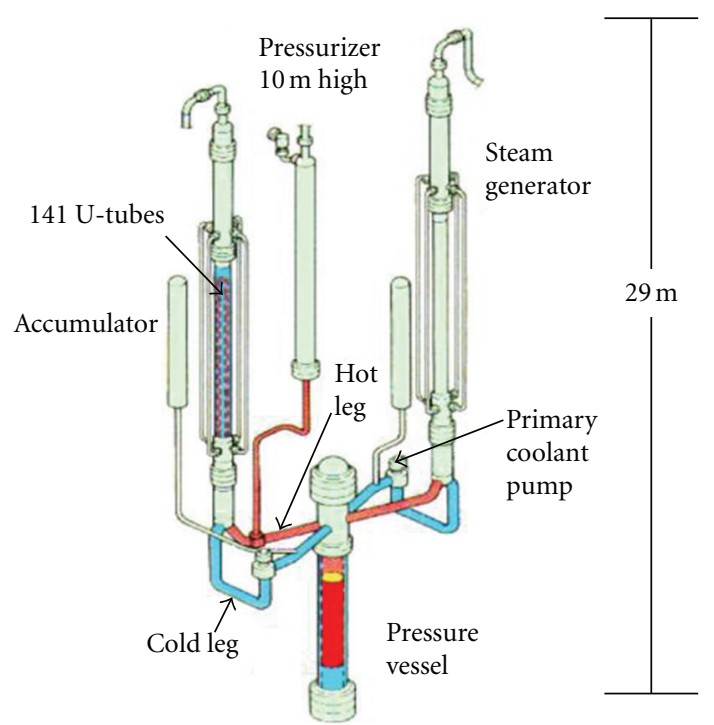

FIgURE 2: Schematic view of ROSA/LSTF.

TABLE 1: Details of LSTF U-tubes in each SG.

\begin{tabular}{lccc}
\hline Type & $\begin{array}{c}\text { Straight } \\
\text { length }(\mathrm{m})\end{array}$ & $\begin{array}{c}\text { Number of } \\
\text { tubes }\end{array}$ & Instrumented tubes \\
\hline 1 & 9.44 & 21 & Two short tubes \\
2 & 9.59 & 19 & \\
3 & 9.74 & 19 & \\
4 & 9.89 & 19 & Two medium tubes \\
5 & 10.04 & 17 & \\
6 & 10.19 & 15 & \\
7 & 10.34 & 13 & Two long tubes \\
8 & 10.49 & 11 & \\
9 & 10.64 & 7 & \\
\hline
\end{tabular}

U-tubes (inner diameter of $19.6 \mathrm{~mm}$, nine different heights as shown in Table 1), inlet and outlet plena, boiler section, steam separator, steam dome, steam dryer, main steam line, four downcomer pipes, and other internals. Six U-tubes are instrumented for each SG. Instrumented tubes designated as Tubes 1 and 6 are short tubes (Type 1 in Table 1), Tubes 2 and 5 are medium tubes (Type 5), and Tubes 3 and 4 are long tubes (Type 9). The hot and cold legs, $207 \mathrm{~mm}$ in inner diameter, are sized to conserve the volumetric scaling (2/48) and the ratio of the length to the square root of the diameter to simulate the flow regime transitions in the horizontal legs [9]. All types of ECCS are equipped with additional features such as ECCS coolant temperature control especially for accumulators.

The LSTF core, $3.66 \mathrm{~m}$ in active height, consists of 1008 electrically heated rods in 24 rod bundles to simulate the fuel rod assembly in the reference PWR. Axial core power profile is a 9-step chopped cosine with a peaking factor of 1.495. The radial power profile is achieved by providing three different power bundles (high, mean, and low) with a maximum peaking factor of 1.51 for high-power bundle. The LSTF initial core power of $10 \mathrm{MW}$ corresponds to $14 \%$

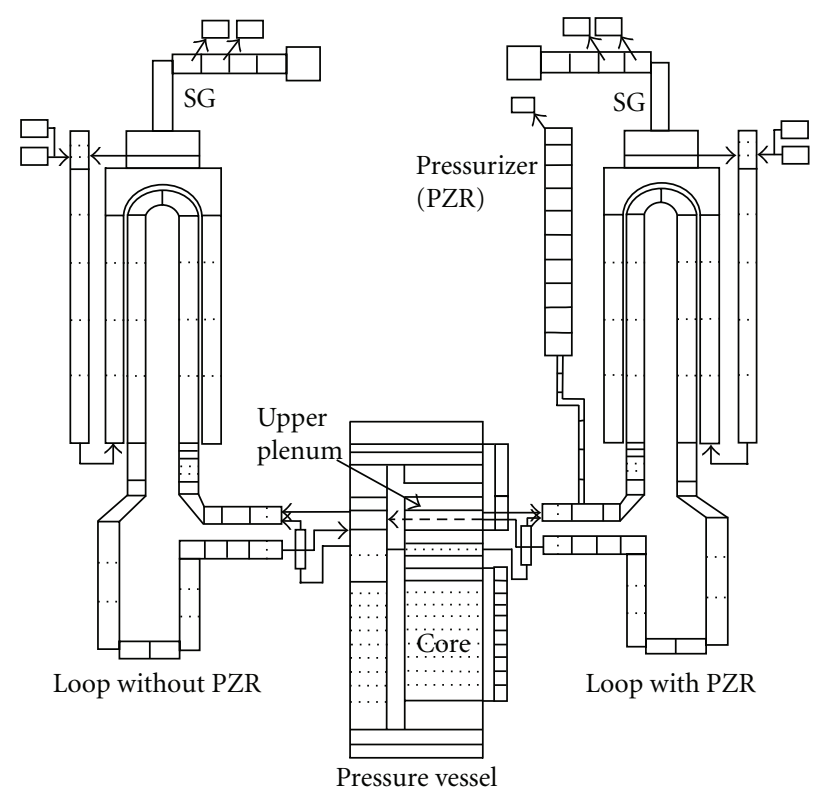

Figure 3: Noding schematic of PWR for RELAP5 analysis.

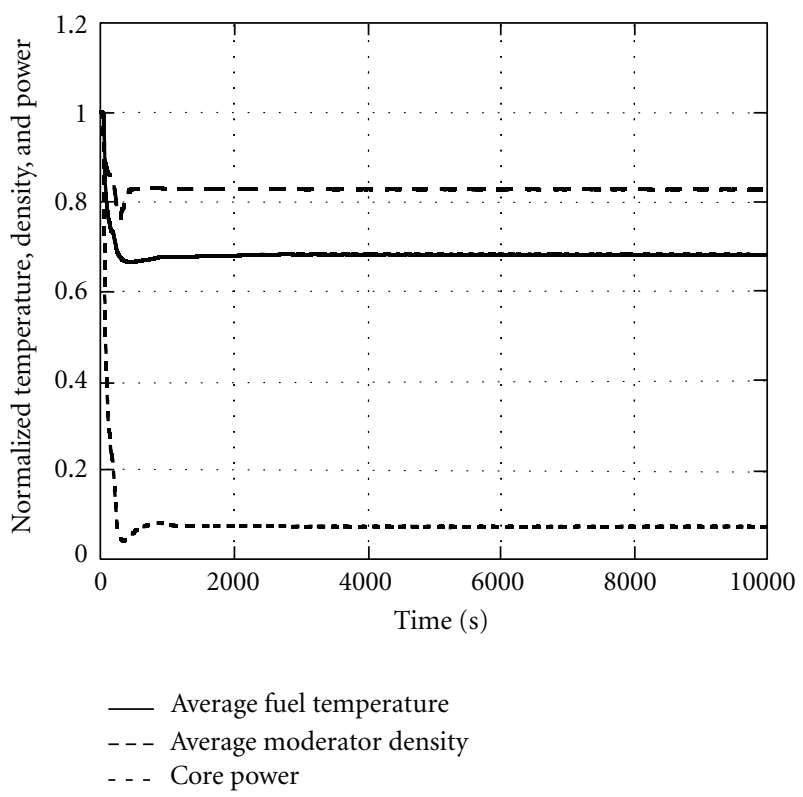

FIGURE 4: PWR average fuel temperature, average moderator density, and core power calculated by RELAP5 code.

of the volumetric-scaled (1/48) PWR nominal core power because of a limitation in the capacity of power supply. The core power after the test initiation is then kept constant at $10 \mathrm{MW}$ for a little while before the core power starts to follow predetermined decay curve.

Instrumentations around 1900 channels provide detailed information on thermal-hydraulic conditions and responses for such parameters as temperature, liquid level, pressure, flow rate, and fluid density. The break flow rate including discharge flow rate through PORV of PZR is estimated from the level increase rate in the catch tank called ST. Visual 


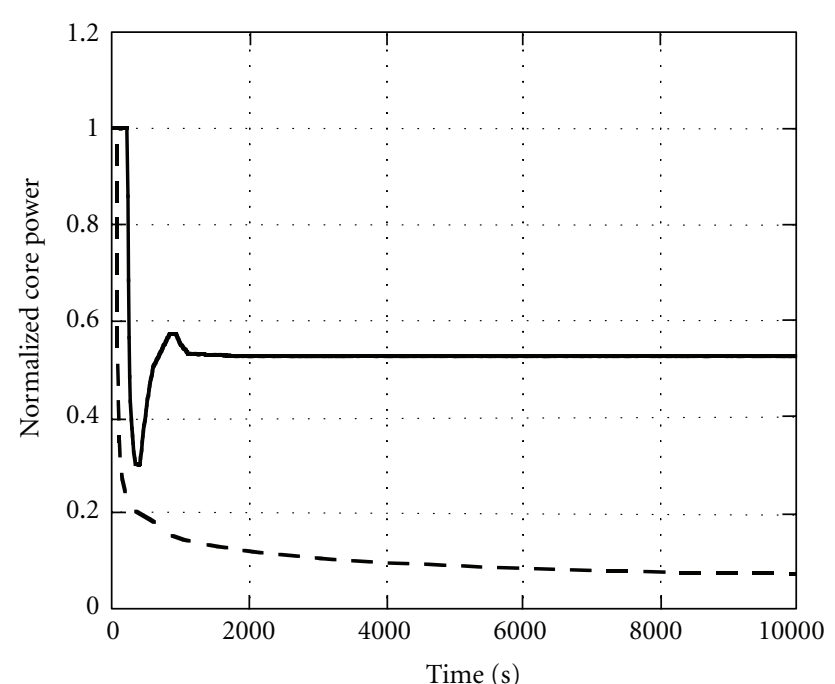

_ Predetermined LSTF core power

- - - LSTF core power in LOFW with scram

FIGURE 5: LSTF core power curve pre-determined through PWR analysis, being compared with LSTF core power decay curve in case of LOFW with scram.

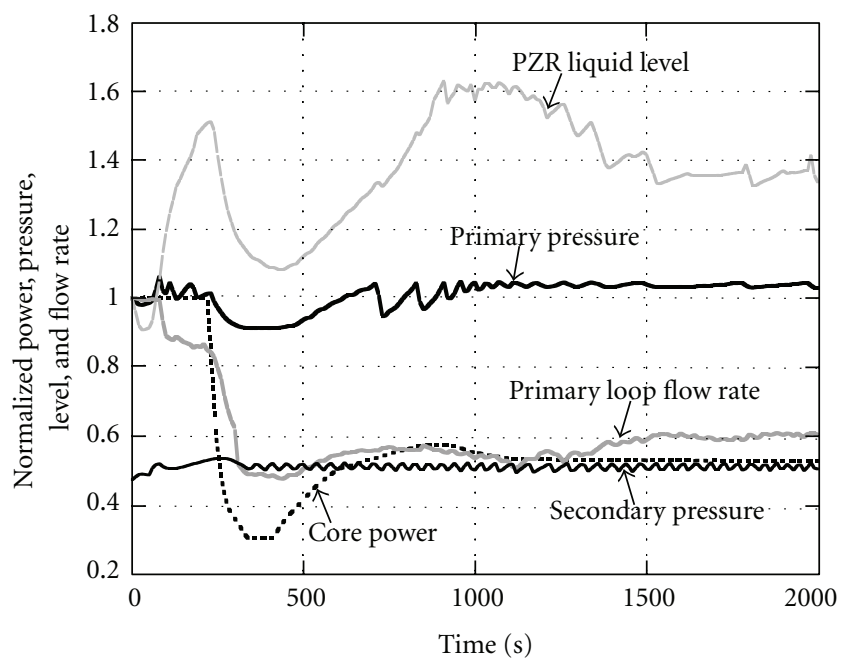

FIGURE 6: LSTF results for core power, primary and secondary pressures, PZR liquid level, and primary mass flow rate in loop with PZR (0 to $2000 \mathrm{~s})$.

observation of flow is conducted in horizontal legs by using "video probe," a periscope that withstands high-temperature steam/water conditions employing cooling-free glass-fiber cables or air-cooled bore scopes.

Since the shakedown test in 1985, the LSTF has provided experimental data including the 5\% cold leg break LOCA simulation for OECD/NEA ISP-26 and the Mihama Unit-2 SG tube rupture accident for the Nuclear Safety Commission of Japan. Throughout its operational history, the facility has demonstrated excellent experimental capabilities and

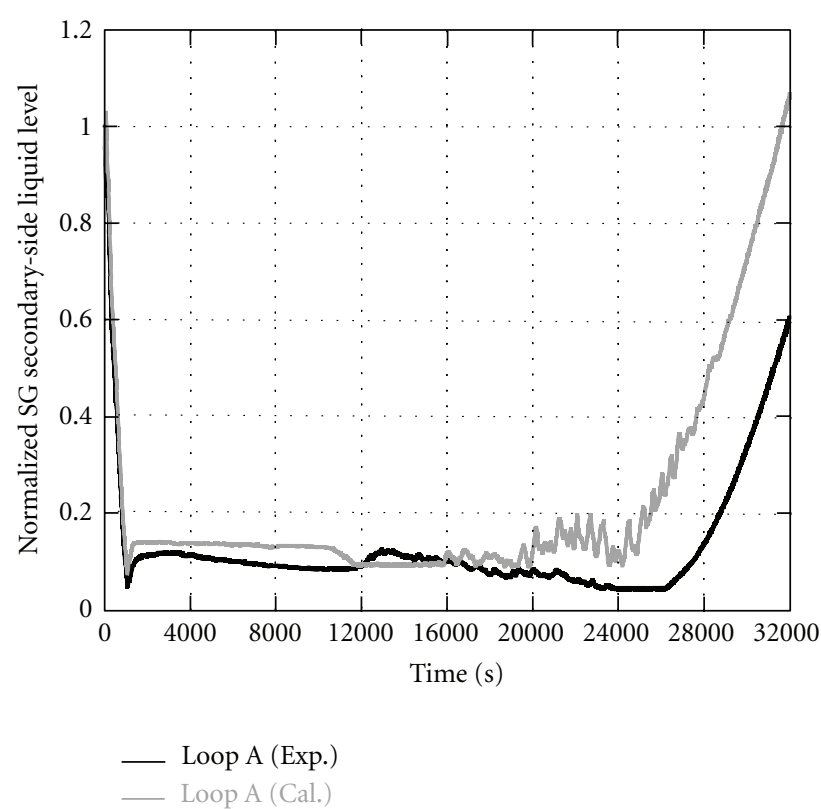

Figure 7: LSTF and RELAP5 results for SG secondary-side collapsed liquid level in loop with PZR.

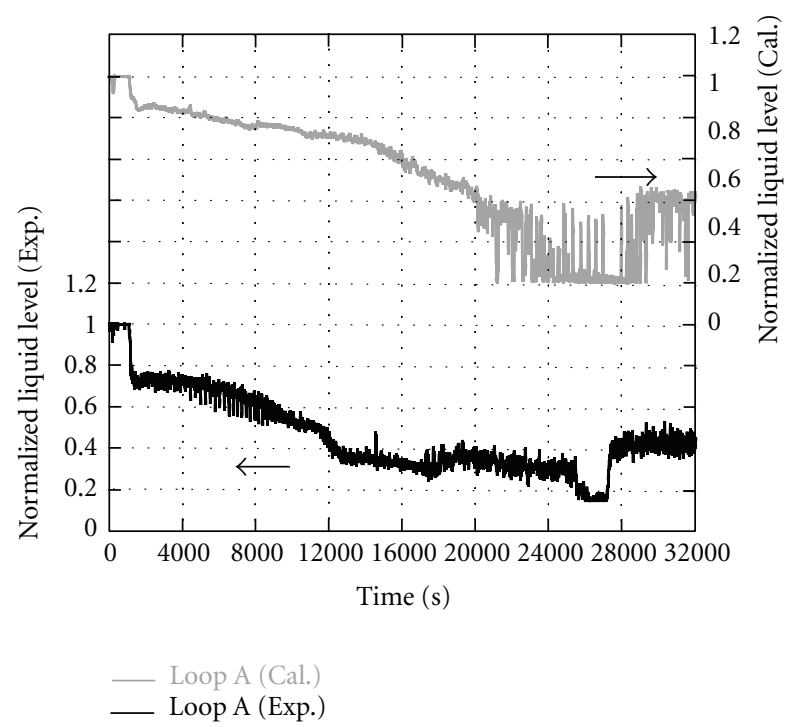

FIGURE 8: LSTF and RELAP5 results for hot leg liquid level in loop with PZR.

has provided unique data for nonequilibrium, nonhomogeneous, and multidimensional phenomena that may arise during reactor accidents and abnormal transients.

\section{PWR Analysis to Define LSTF Core Power}

Analysis for PWR LOFW transient without scram was performed by the RELAP5/MOD3.2.1.2 code to define the LSTF core power to correctly simulate the power transient under influences of coolant conditions such as density and 


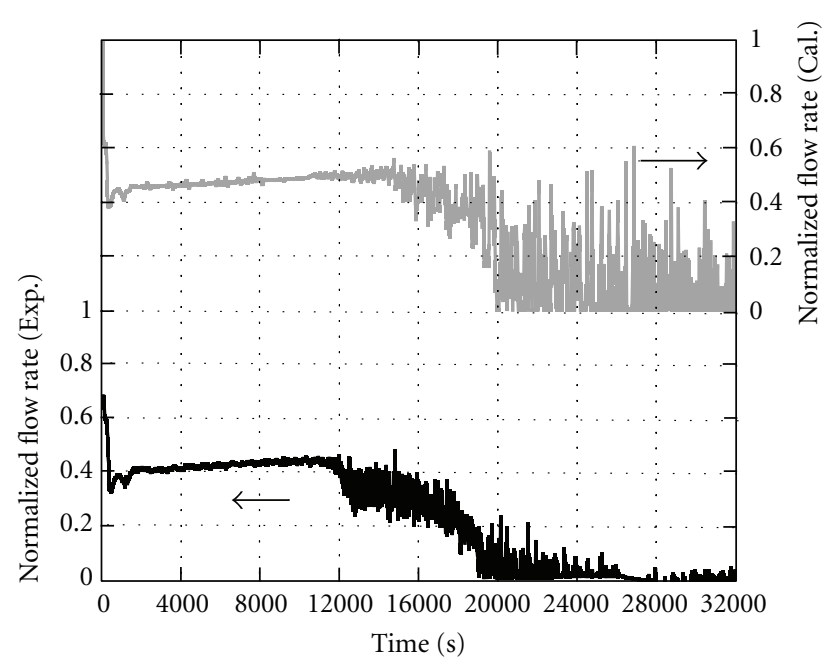

L Loop A (Cal.)

FIGURE 9: LSTF and RELAP5 results for primary mass flow rate in loop with PZR.

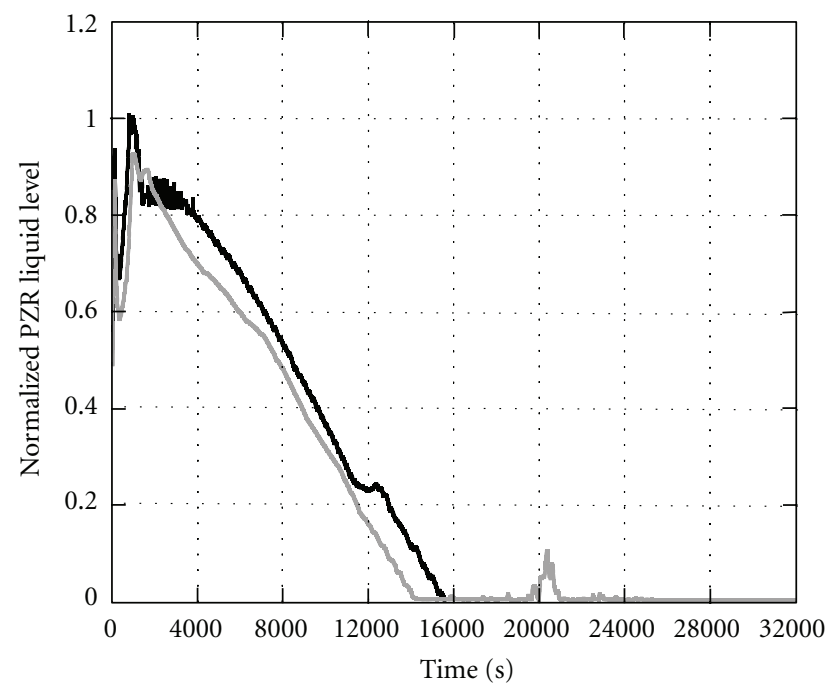

_ Exp.<smiles>C=[14CH2]</smiles>

FIGURE 10: LSTF and RELAP5 results for PZR liquid level.

void fraction in the core. Figure 3 shows a noding schematic of PWR for the RELAP5 analysis. PWR system is modeled in one-dimensional manner including pressure vessel, primary loops, PZR, SGs, and SG secondary-side system. Four-loop PWR is then modeled by a two-loop noding, similar to that for LSTF noding (to be shown in Figure 23), which is composed of one loop with PZR and the other loop corresponding to three loops without PZR. The Doppler and moderator density reactivity coefficients are based on fourloop PWR reference data at the beginning of cycle under the hot nominal core power condition [7]. The PWR analysis

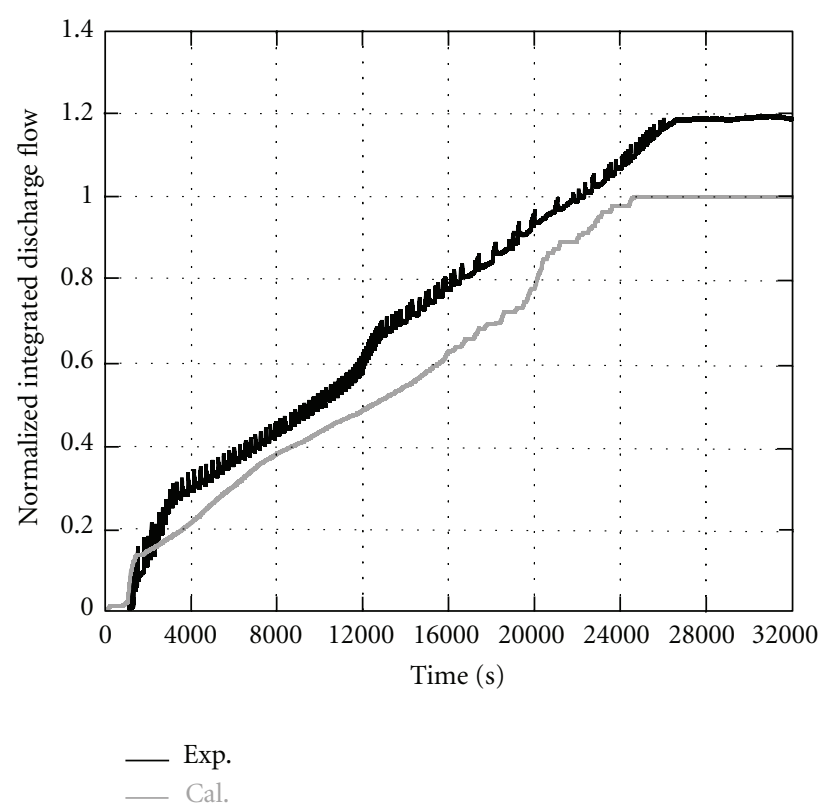

FIGURE 11: LSTF and RELAP5 results for integrated discharge flow through PORV.

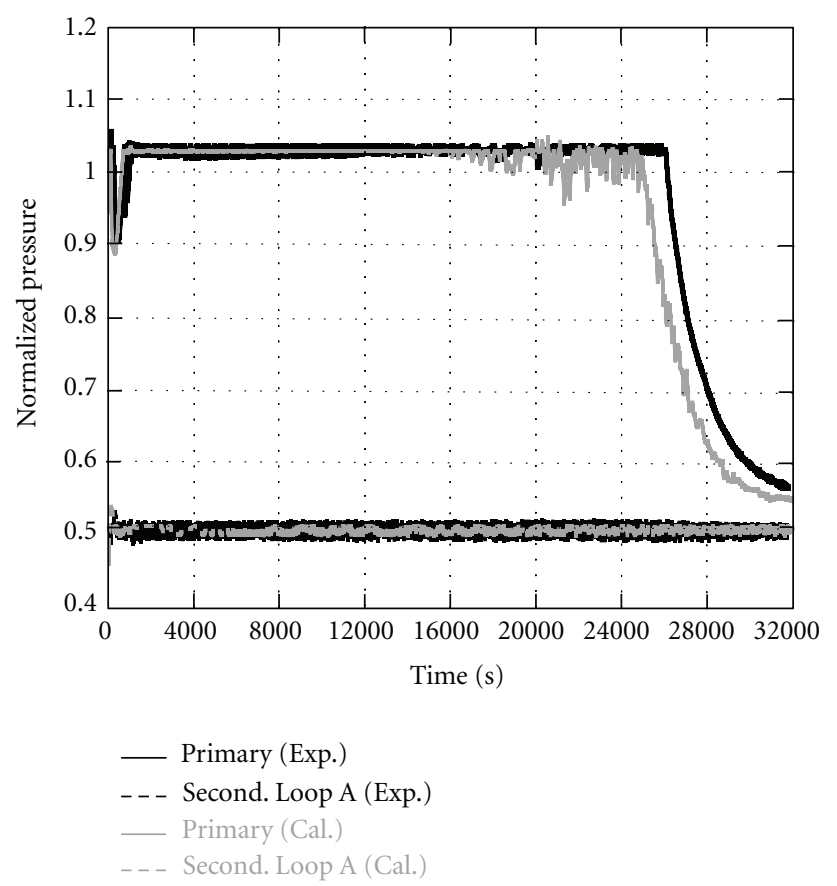

FIGURE 12: LSTF and RELAP5 results for primary and secondary pressures in loop with PZR.

employed RELAP5 critical flow model for the PORV with a discharge coefficient of 1.0. Major assumptions include total failure of HPI system and loss of off-site power at scram signal.

"SG secondary-side narrow-range liquid level low" signal is generated to trigger scram signal. The set point of this signal is $10 \%$ of the SG secondary-side narrow-range liquid 


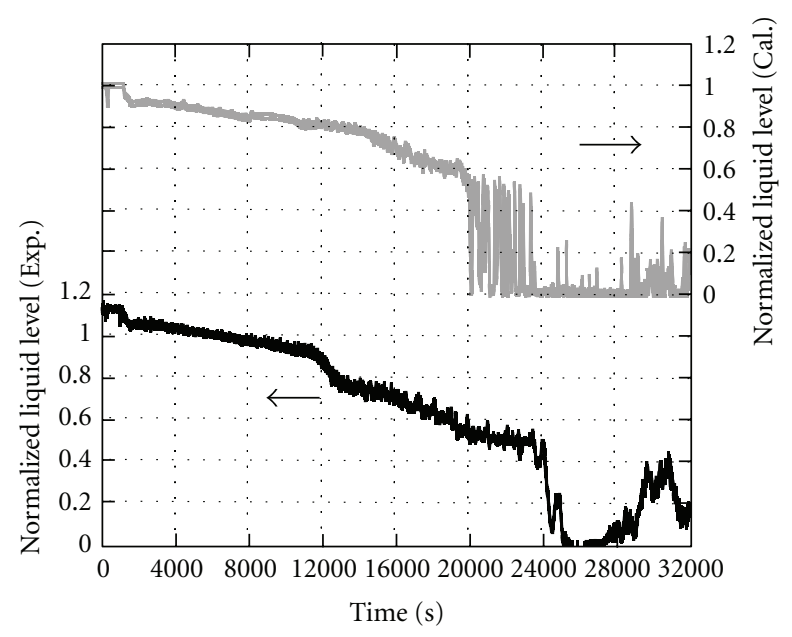

Loop A (Cal.)
Loop A (Exp.)

FIGURE 13: LSTF and RELAP5 results for SG inlet plenum collapsed liquid level in loop with PZR.

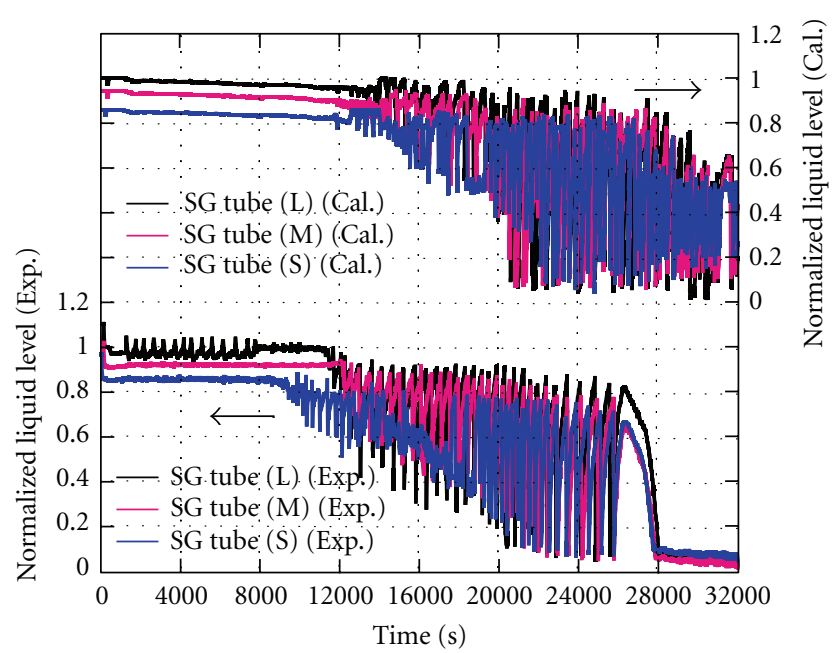

FIGURE 14: LSTF and RELAP5 results for collapsed liquid levels in upflow side of SG U-tubes in loop with PZR.

level. The scram signal was generated at $50 \mathrm{~s}$, causing the closure of SG main steam isolation valves (MSIVs) and the coastdown of primary coolant pumps. This condition was employed in the LSTF experiment. AFW in both loops was assumed to start when the SG secondary-side becomes empty of liquid.

Figure 4 compares the calculated average fuel temperature, average moderator density, and core power. The core power decreased due to negative feedback induced mainly by an increase in the average fluid temperature, thus a decrease in the average coolant density. The core power was kept at around $250 \mathrm{MW}(7.4 \%)$ after about $1100 \mathrm{~s}$ due to the balance of Doppler and moderator density reactivity, reaching almost nearly equilibrium conditions. The core power curve obtained by the RELAP5 code analysis was

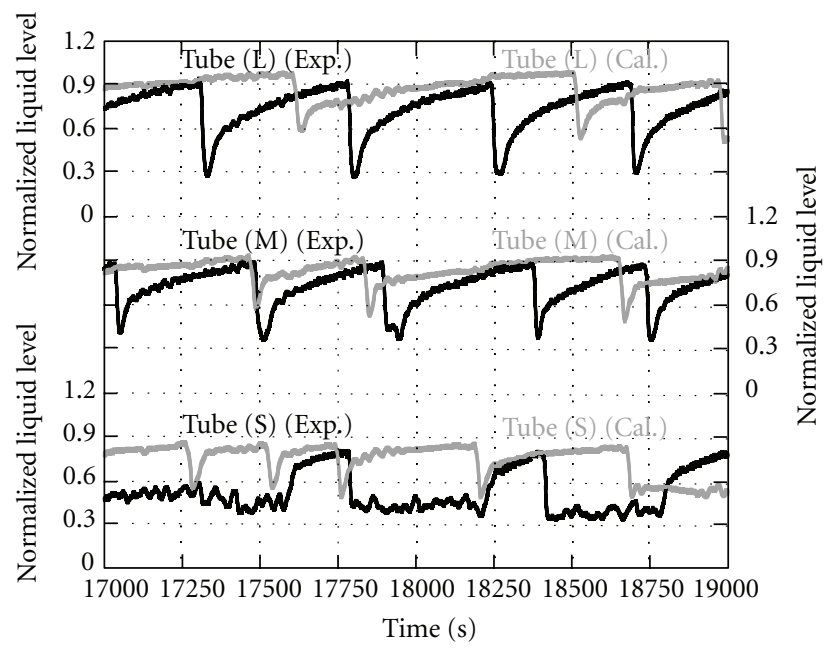

FIGURE 15: LSTF and RELAP5 results for collapsed liquid levels in upflow side of SG U-tubes in loop with PZR during large-amplitude level oscillation period.

referred in the LSTF experiment. Figure 5 shows the predetermined core power curve obtained from the PWR analysis and consideration of volumetric scaling of LSTF, being compared with LSTF core power decay curve in case of LOFW with scram [10]. The portion higher than $10 \mathrm{MW}$ $(14 \%)$ is cut off due to the limitation in the power supply.

\section{LSTF Experiment and Code Analysis Results}

5.1. LSTF Test Conditions. The PZR pressure, fluid temperatures in hot and cold legs during initial steady-state conditions were $15.5 \mathrm{MPa}, 597 \mathrm{~K}$, and $563 \mathrm{~K}$, respectively, according to the reference PWR conditions. Initial secondary pressure was raised to $7.3 \mathrm{MPa}$ from nominal value of 6.1 MPa to limit the primary-to-secondary heat transfer rate to $10 \mathrm{MW}$. Set point pressures for opening and closure of the PZR PORV are 16.20 and $16.07 \mathrm{MPa}$, respectively, and are 8.03 and $7.82 \mathrm{MPa}$ for the SG relief valves (RVs), referring to the corresponding values in the reference PWR. Initial SG secondary-side collapsed liquid level was set to about $7.3 \mathrm{~m}$ based on the PWR analysis result, for better simulation of the liquid level transient in the reference PWR. To avoid deformation of SG structures including U-tubes (bottom) due to thermal shock from injection of cool AFW coolant onto high-temperature structures, AFW was initiated in both loops when the SG secondary-side collapsed liquid level still remained by at least about $0.5 \mathrm{~m}$. AFW flow rate in each loop was fixed to about $0.85 \mathrm{~kg} / \mathrm{s}$, which corresponded to about $120 \%$ of the volumetrically scaled rate, to maintain a continuous primary-to-secondary heat removal under high core power through the LSTF pre-test analysis [11].

5.2. Major Phenomena Observed in the Experiment. The LSTF test results are shown in Figures 7-21 as a comparison with RELAP5 posttest analysis results, except for Figure 16. The major phenomena observed in the experiment are 


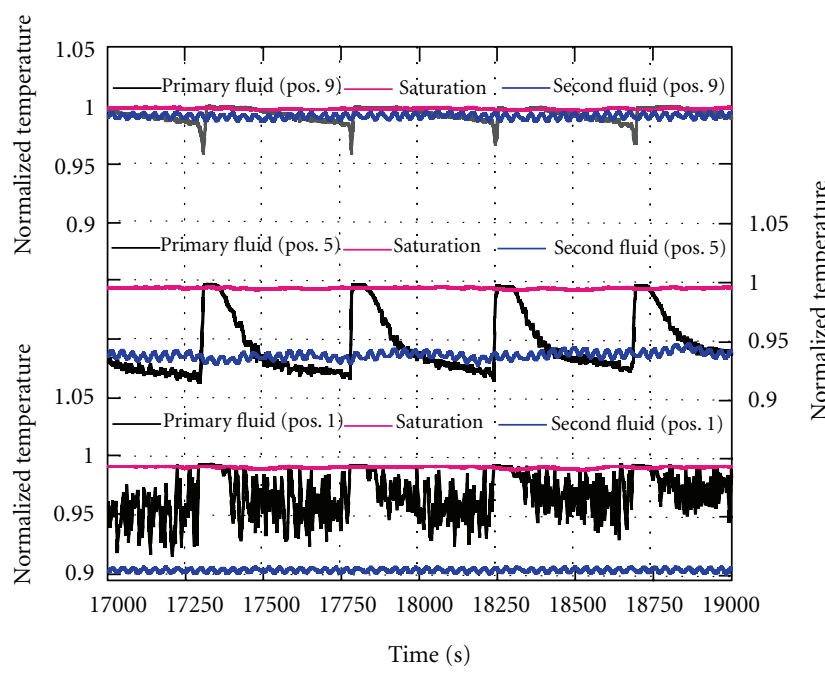

(a) Tube upflow side

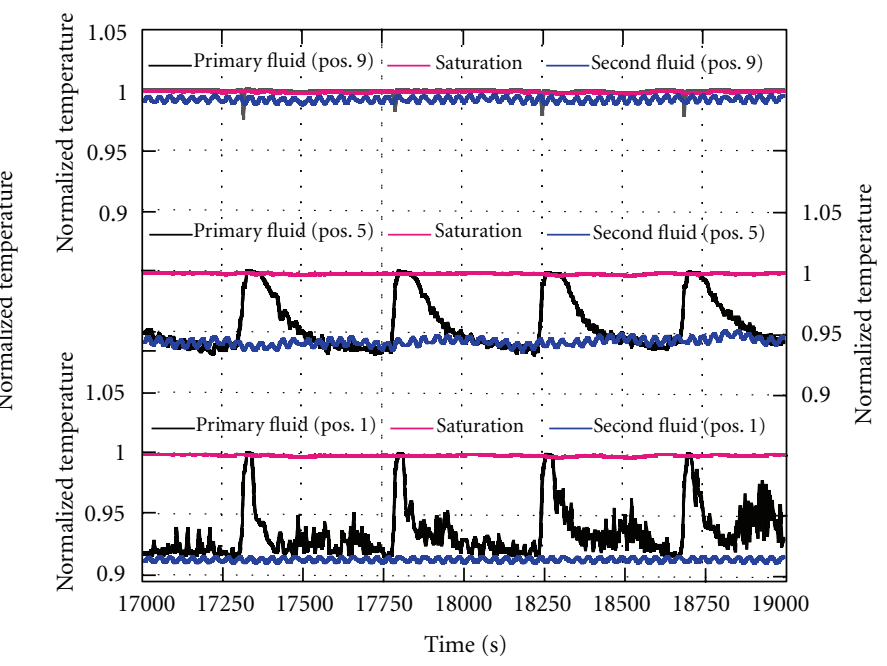

(b) Tube downflow side

FIGURE 16: LSTF results for fluid temperatures in primary and secondary side of SG Tube 3 in loop with PZR at Positions 1, 5 and 9 during large-amplitude level oscillation period.

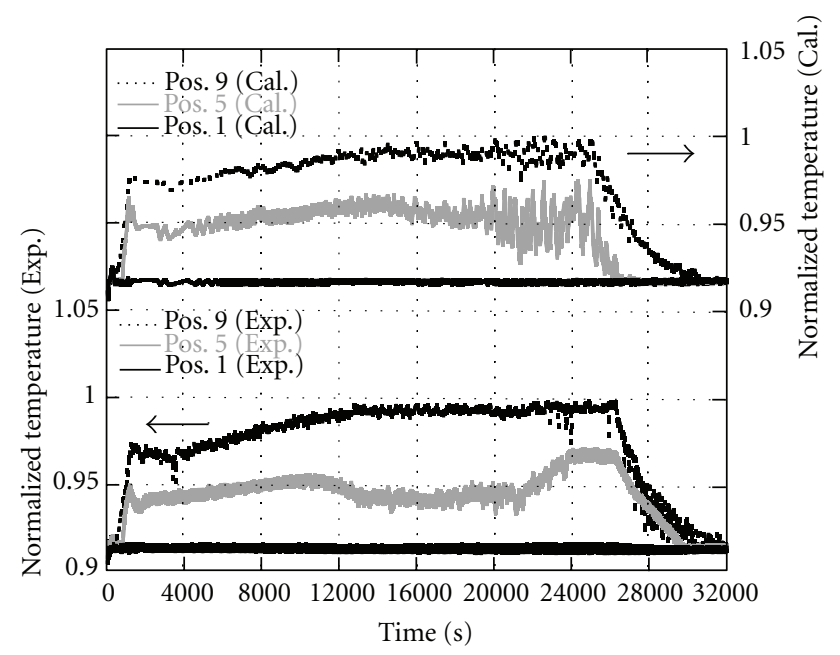

Figure 17: LSTF and RELAP5 results for fluid temperatures in secondary-side of SG Tube 3 in loop with PZR at Positions 1, 5, and 9.

explained by classifying them into the following three stages of transient.

(1) Before Initiation of AFW (0 to about 1030 s). The experiment was initiated by the termination of main feedwater at time zero. The core power was maintained at $10 \mathrm{MW}$ for $170 \mathrm{~s}$ after the scram signal, while it was kept at around $5.3 \mathrm{MW}(7.4 \%)$ after about $1100 \mathrm{~s}$ as shown in Figure 5 until automatic power reduction due to high core temperature.

Figure 6 compares major parameters in the initial transient. After the MSIV closure at about 55 s, the SG secondaryside pressure increased rapidly up to about $8.4 \mathrm{MPa}$, causing the SG RV to be kept opened for a while due to high core power. The primary pressure also increased rapidly after the MSIV closure up to around $16 \mathrm{MPa}$ due to high core power and oscillated thereafter by cycle opening of the PORV. The PZR liquid level continuously increased after the MSIV closure irrespective of cycle opening of the PORV, except some while after about $230 \mathrm{~s}$ in response to the core power decrease thus the primary pressure decrease. The PZR became full of liquid temporarily after about $900 \mathrm{~s}$ by volumetric expansion of coolant. Single-phase liquid natural circulation started at about $300 \mathrm{~s}$ when the primary coolant pumps stopped. The SG secondary-side collapsed liquid level rapidly decreased to about $0.5 \mathrm{~m}$ at about $1030 \mathrm{~s}$ as shown in Figure 7, and the AFW was started the coolant injection in both SGs.

(2) After Initiation of AFW (about 1030 to $24000 \mathrm{~s}$ ). The primary loop flow turned into two-phase natural circulation after about $1150 \mathrm{~s}$ when liquid level appeared in the hot leg as shown in Figure 8. The primary loop flow rate gradually increased thereafter as shown in Figure 6 and started to decrease with some oscillation after about $11500 \mathrm{~s}$ as shown in Figure 9 when the liquid level in the hot leg became lower than the half height. The PZR liquid level shown in Figure 10 decreased very slowly after the actuation of AFW. The PZR liquid level stayed at certain constant levels of about $83 \%$ at around $1500-3500 \mathrm{~s}$ and of about $23 \%$ at around 11500-12500 s probably due to flooding at the PZR bottom because of temporary high discharge rate through the PORV as shown in Figure 11. The PZR became empty of liquid at about $15800 \mathrm{~s}$.

AFW initiated at about $1030 \mathrm{~s}$ provided a continuous primary-to-secondary heat removal. The SG secondary-side pressure was kept at around $8 \mathrm{MPa}$ by frequent cycle opening of the RVs as shown in Figure 12. There was no apparent relationship between the cycle open timings of the PORV and the SG RVs. 


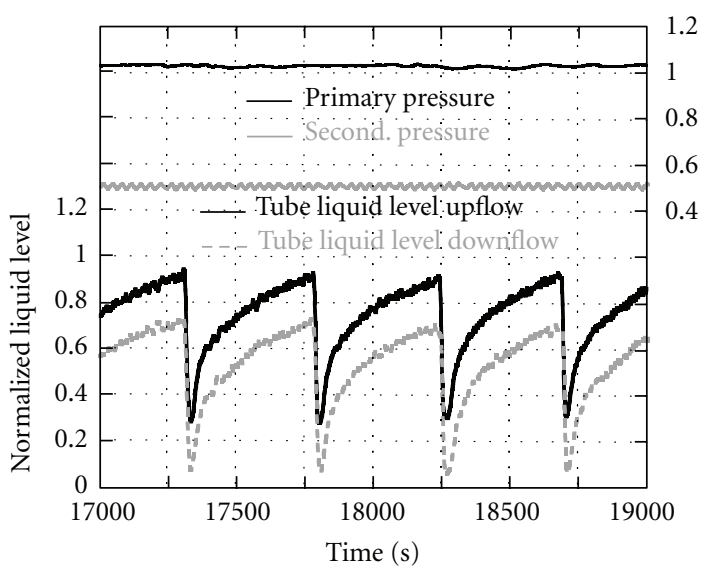

(a) LSTF result (17000 to $19000 \mathrm{~s}$ )

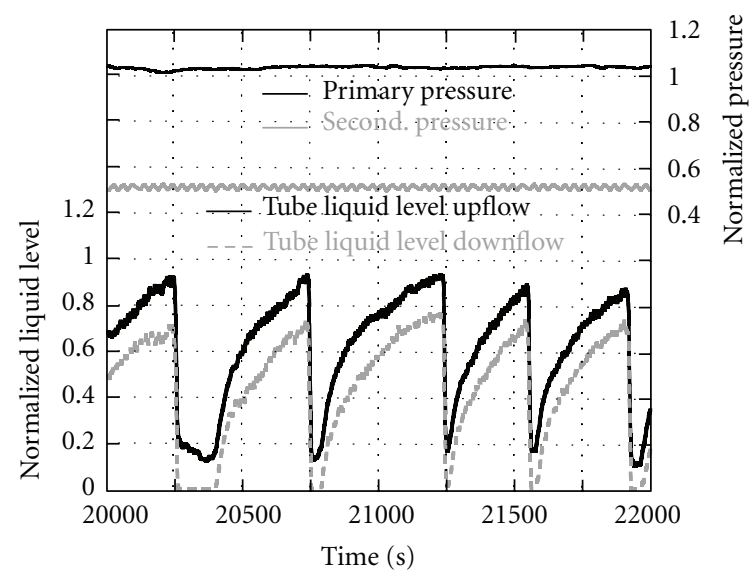

(c) LSTF result (20000 to $22000 \mathrm{~s}$ )

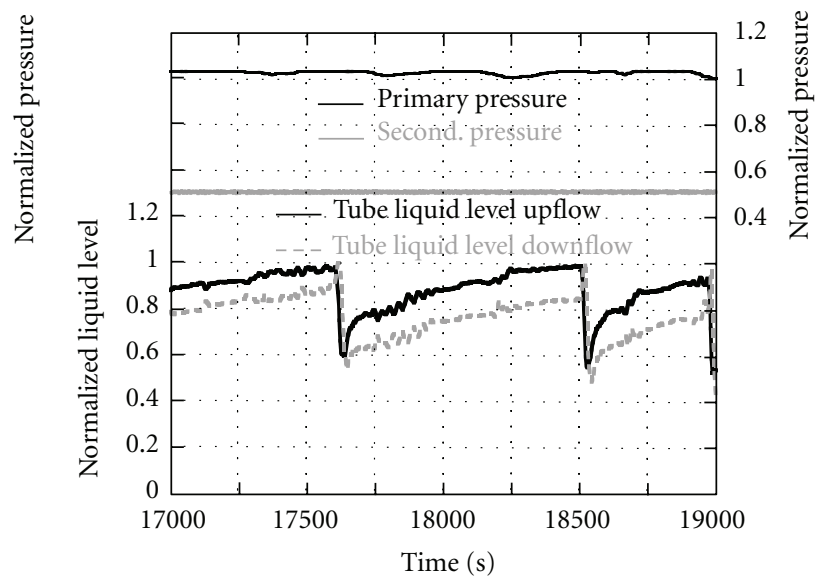

(b) RELAP5 result (17000 to $19000 \mathrm{~s}$ )

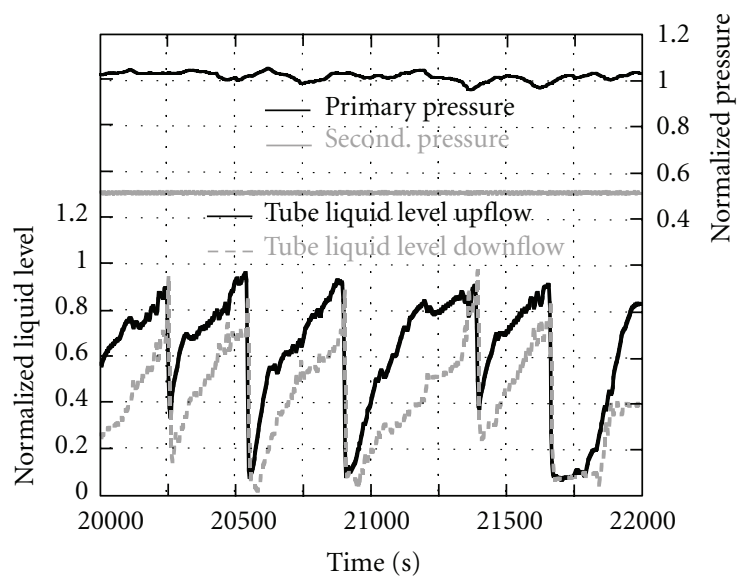

(d) RELAP5 result (20000 to $22000 \mathrm{~s}$ )

FIGURE 18: LSTF and RELAP5 results for collapsed liquid levels in SG Tube 3 in loop with PZR compared with primary and secondary pressures during large-amplitude level oscillation period.

Figures 13 and 14, respectively, show the collapsed liquid levels in SG inlet plenum and upflow side of SG U-tubes, typically Tube 1 designated as $\mathrm{S}$, Tube 2 designated as $\mathrm{M}$, and Tube 3 designated as L of SG in the loop with PZR (loop A). The void fraction in the SG inlet plenum gradually increased, but SG U-tubes were filled almost with singlephase liquid until around $11500 \mathrm{~s}$. After the voiding started in the SG U-tubes, the primary loop flow rate shown in Figure 9 gradually decreased as the void fraction increased in the U-tubes. A large-amplitude long-term oscillation developed in the void fraction in each of U-tubes rather randomly as shown in Figure 15. Large-amplitude level oscillation, in a form of slow fill and dump, started after around 10200, 12000, and $11400 \mathrm{~s}$, respectively, in Tubes S, M, and L in the loop with PZR. The onset timings were different among U-tubes but similar in the two instrumented tubes with the same length. The time period of the fill and dump depended on the tube length such that the period is longer in the longer tubes. Integration of such a random fill and dump behavior in many SG U-tubes resulted in the continuous natural circulation through the loop with rather small flow fluctuation.
Figure 16 shows the fluid temperatures in both the primary and secondary side of SG Tube 3 in the loop with PZR at Positions 1, 5, and 9, respectively, at about 0.8, 3.4, and $8.5 \mathrm{~m}$ from the bottom of SG boiler section during the large-amplitude level oscillation period. The primary saturation temperature based on the upper plenum pressure is compared as a reference. There was fluid temperature gradient in the secondary side as the temperature increased with the elevation. The primary fluid temperature at Position 9 stayed almost at the saturation temperature except when the dump phenomena (drain of low-temperature condensate column to downflow side) happened, indicating small steam heat removal to the secondary side thus less steam condensation. At the lower elevations, the primary fluid temperature fluctuated between the primary saturation and secondary-side temperatures. During the long-term fill period when the low-temperature condensate column develops, the primary coolant temperature in the upflow side became even lower than the secondary-side fluid temperature typically at Position 5 because low-temperature coolant rose from the lower elevation with lower secondaryside fluid temperature. At the entrance of the tube, saturated 


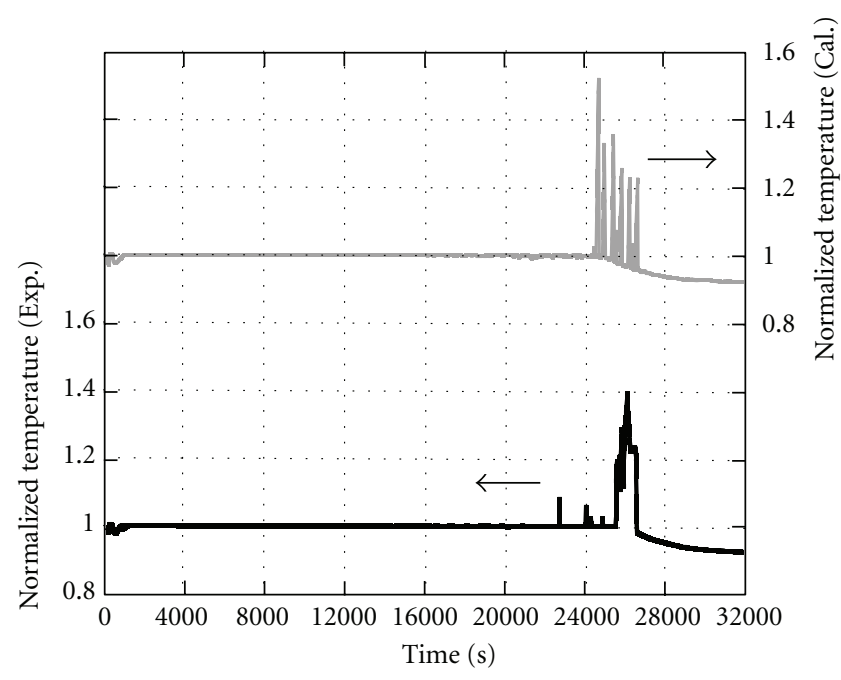

Pos. 7 temp. (Cal.)
— Pos. 7 temp. (Exp.)

FIGURE 19: LSTF and RELAP5 results for cladding surface temperature.

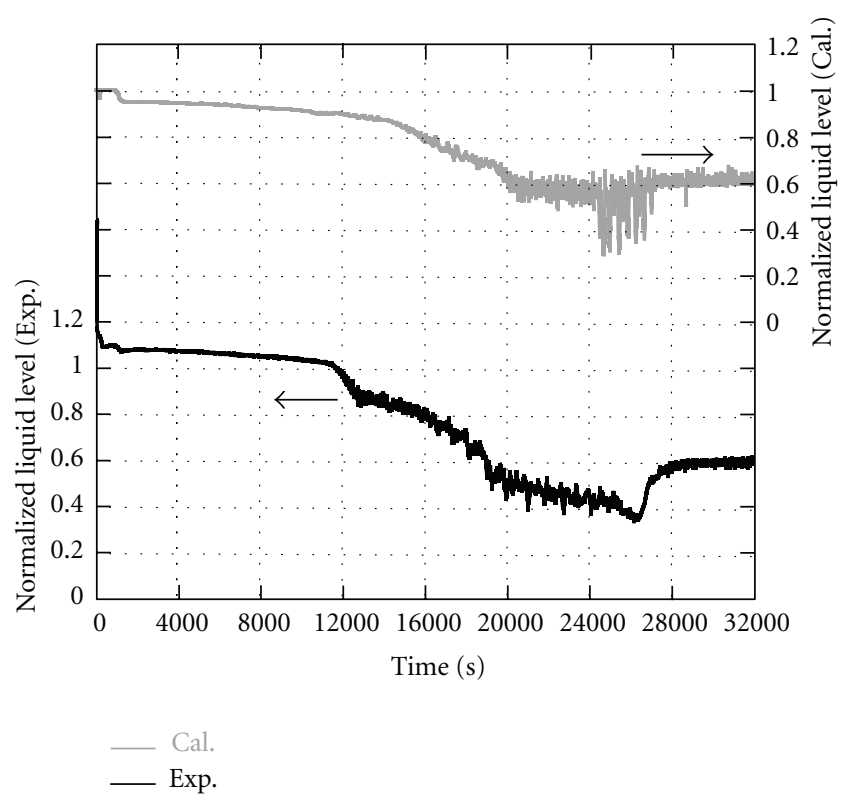

FIGURE 20: LSTF and RELAP5 results for core collapsed liquid level.

steam constantly entered probably as two-phase mixture, causing frequent temperature fluctuation. The primary fluid temperature in the downflow-side, on the other hand, stayed at the secondary-side fluid temperature and recovered to the primary saturation temperature only when the water column drains, because the SG outlet plenum was filled with subcooled coolant. Large temperature difference as much as $54 \mathrm{~K}$ between primary and secondary coolant at around the tube entrance enhanced the effective steam condensation. The SG secondary-side fluid temperature at Position 1 stayed almost at certain temperature as shown in Figure 17.

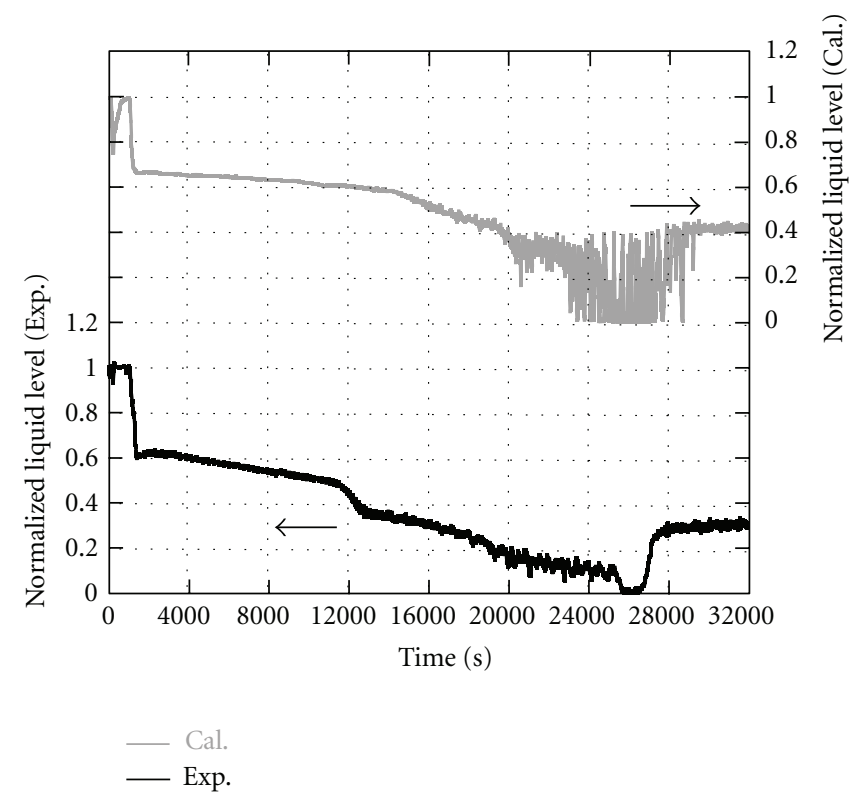

FIGURE 21: LSTF and RELAP5 results for upper plenum collapsed liquid level.

Figure 18 shows the collapsed liquid levels in SG Tube 3 in the loop with PZR during the large-amplitude level oscillation period, being compared with the primary and secondary pressures. Liquid levels slowly increased and suddenly dropped simultaneously in both the upflow side and downflow side of $\mathrm{U}$-tube as the developed water column drained to the SG outlet plenum. This response appeared rather randomly at different timings among the U-tubes (Figure 15). The frequency of the water column development, thus the fill and dump frequency, was far lower than that of the cycle opening of the PORV and the SG RVs.

Such coolant behaviors in the SG U-tubes also appeared in LSTF experiment simulating two-phase natural circulation at high pressures under the constant secondary-side fluid temperature [12]. Intervals between the dumps in the tube with the same length in this experiment, however, were longer than in the natural circulation test due to influences of the vertical fluid temperature distribution along the SG boiler section shown in Figure 17.

(3) During Reflux Condensation Mode (about 24000 to 32000 s). Natural circulation mode may have changed into reflux condensation after around $24000 \mathrm{~s}$ when significant drop started in the SG inlet plenum liquid level (Figure 13). The liquid level in the SG U-tube did not decrease to zero (Figure 14) due to significant steam condensation at the tube entrance as well as CCFL till the end of the test.

The natural circulation contributed to maintain core cooling for rather long time as long as the core is covered by coolant even under high core power (Figures 19 and 20). Temporary large drop happened in the core liquid level when the water column developed in the SG U-tubes after about $22780 \mathrm{~s}$ though liquid level was still in the upper plenum (Figure 21), causing an intermittent increase that 


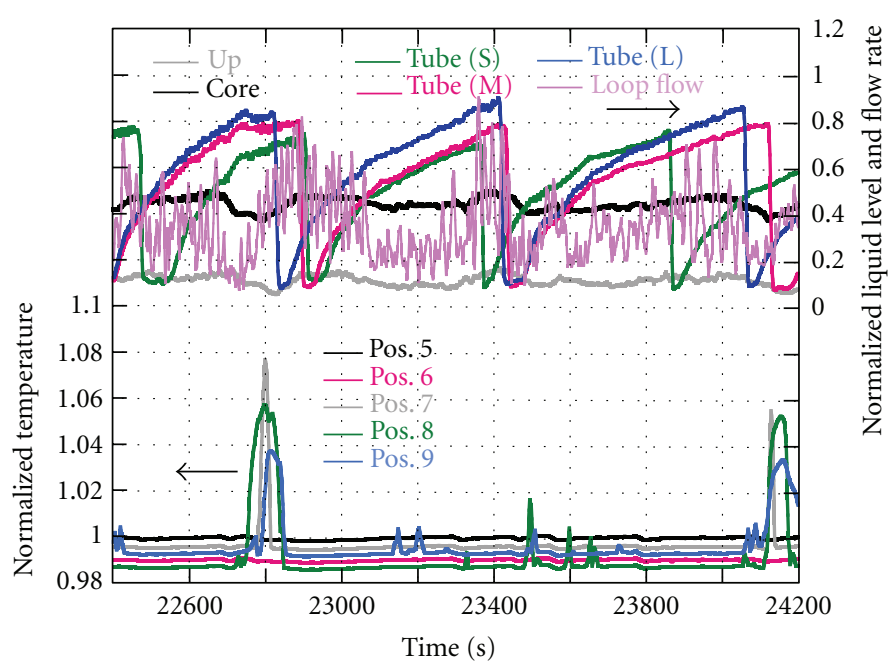

FIGURE 22: LSTF results for cladding surface temperature, collapsed liquid levels in upper plenum, core, and upflow side of SG U-tubes and primary mass flow rate in loop with PZR (22400 to 24200 s).

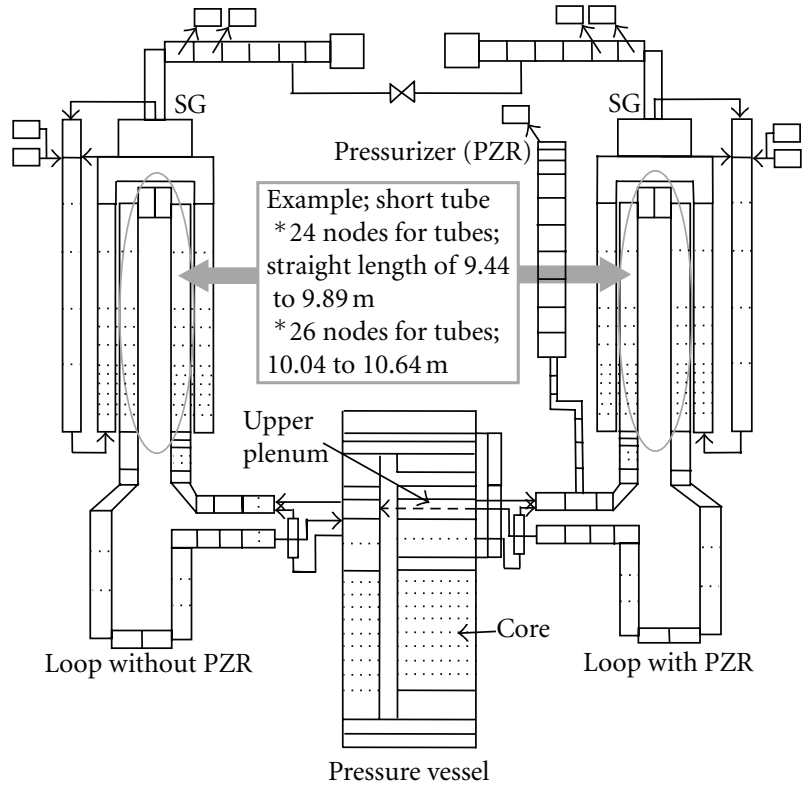

FIGURE 23: Noding schematic of LSTF for RELAP5 analysis.

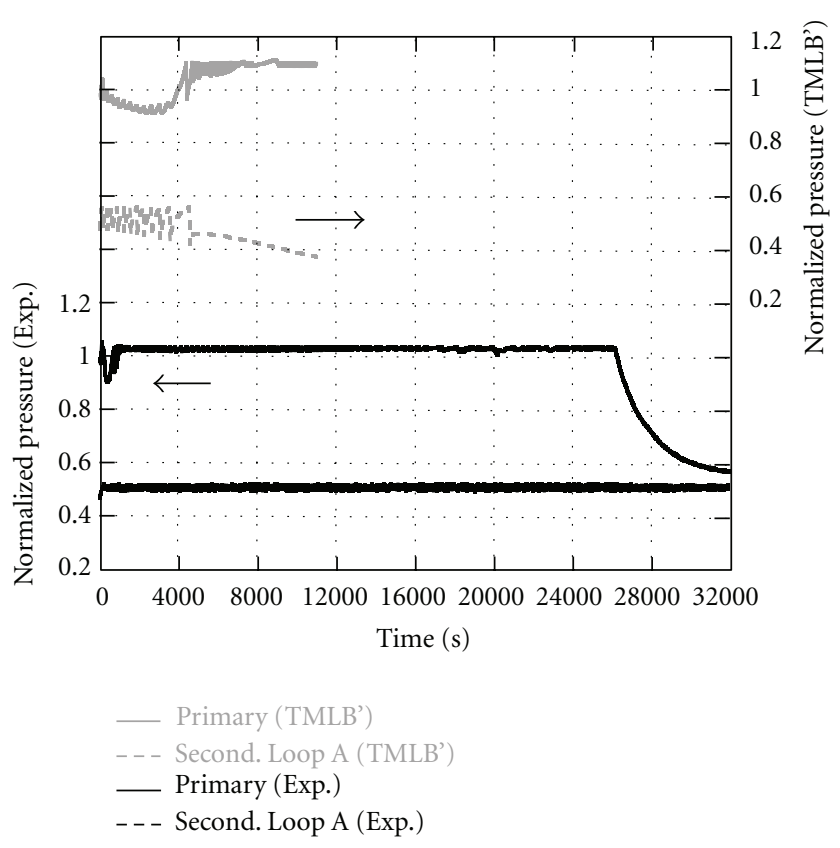

FIgURE 24: Comparison with TMLB' scenario test results for primary and secondary pressures in loop with PZR.

in the secondary-side liquid level (Figure 7). Whole core was quenched, being followed by a decrease in the primary pressure (Figure 12) and an increase in the core liquid level.

\subsection{RELAP5 Code Analysis}

5.3.1. RELAP5 Analysis Conditions. Post-test analyses for the LSTF experiment were conducted with the RELAP5/ MOD3.2.1.2 code by incorporating a two-phase critical flow model [13], which may correctly predict the discharge rate through a sharp-edge orifice to simulate the PORV. The 

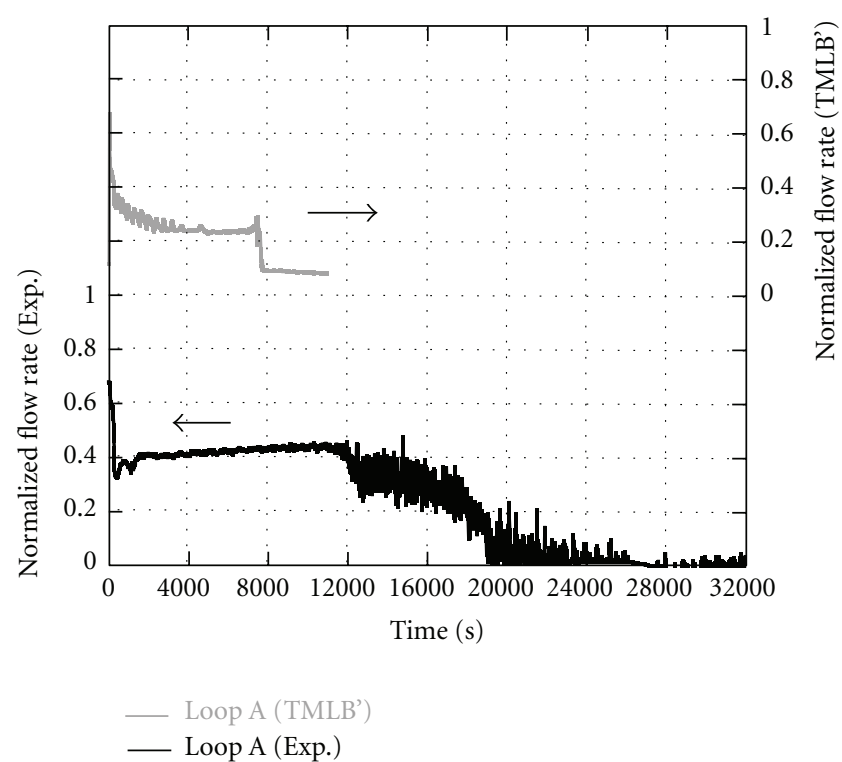

Figure 25: Comparison with TMLB' scenario test results for primary mass flow rate in loop with PZR.
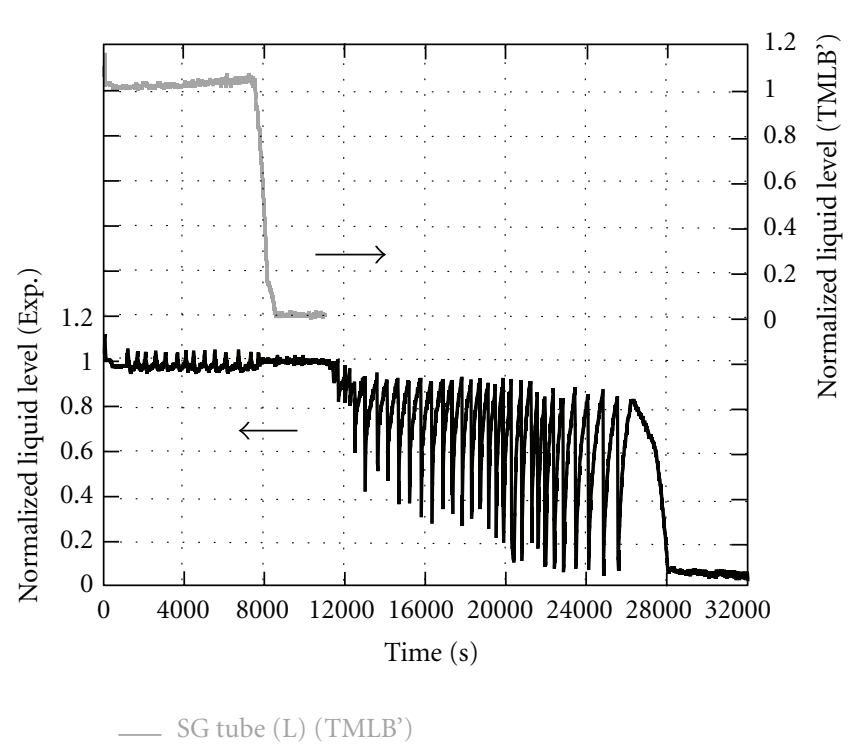

_ SG tube (L) (Exp.)

FIgURE 26: Comparison with TMLB' scenario test results for collapsed liquid level in upflow side of SG Tube 3 in loop with PZR.

model employs the maximum bounding flow theory with a discharge coefficient $(\mathrm{Cd})$ of 0.61 for two-phase discharge flow [14]. Cd of 0.84 was used for single-phase discharge steam [15].

Figure 23 shows a noding schematic of LSTF for RELAP5 analysis. The LSTF system is modeled in one-dimensional manner including pressure vessel, primary loops, PZR, SGs, and SG secondary-side system. The SG U-tubes were modeled by nine parallel flow channels that correspond to 9 types of U-tubes with different height, namely, 24 nodes for short-to-medium tubes (straight length of 9.44 to $9.89 \mathrm{~m}$,

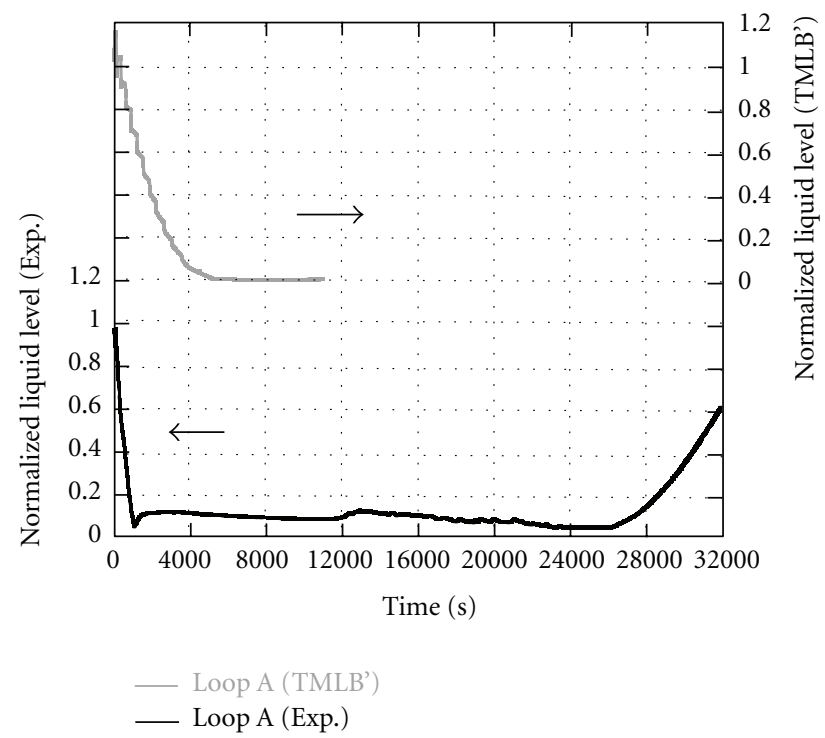

FIGURE 27: Comparison with TMLB' scenario test results for SG secondary-side collapsed liquid level in loop with PZR.

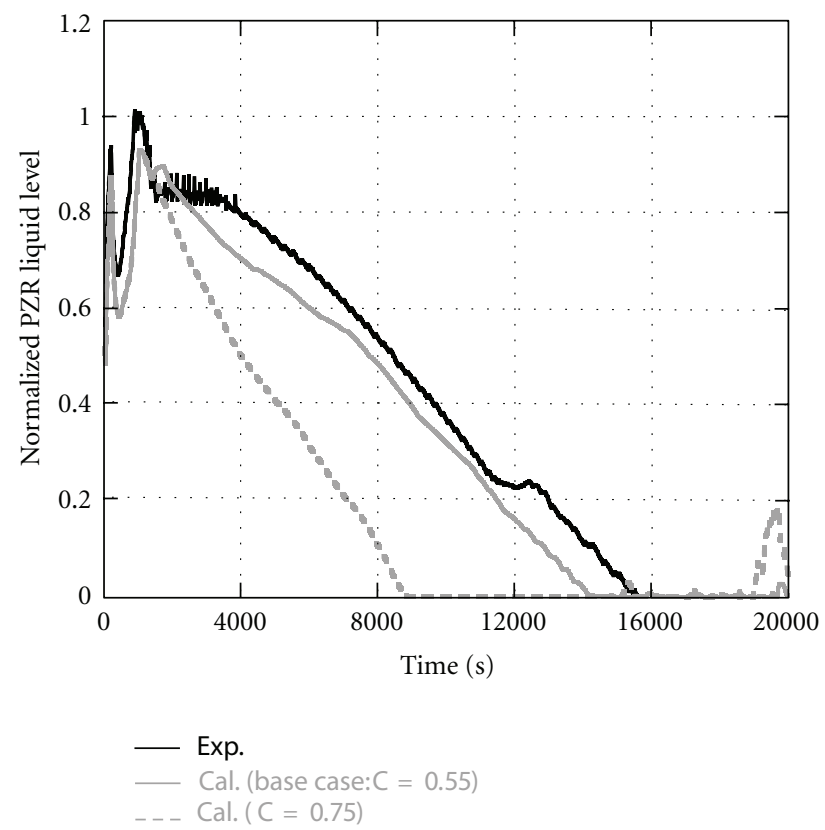

FIGURE 28: RELAP5 results for PZR liquid level in cases with different constants $C$ of Wallis CCFL correlation at both all PZR and surge-line junctions.

four cases in Table 1) and 26 nodes for medium-to-long tubes (straight length of 10.04 to $10.64 \mathrm{~m}$, five cases), for better prediction of the complicated nonuniform coolant behaviors during natural circulation [16].

The core was represented by vertically stacked nine equal-height volumes according to 9-step chopped cosine power profile along the length of the core. The radial power distribution was then given considering the peaking factor and the number of high-, mean- and low-power rod bundles. 

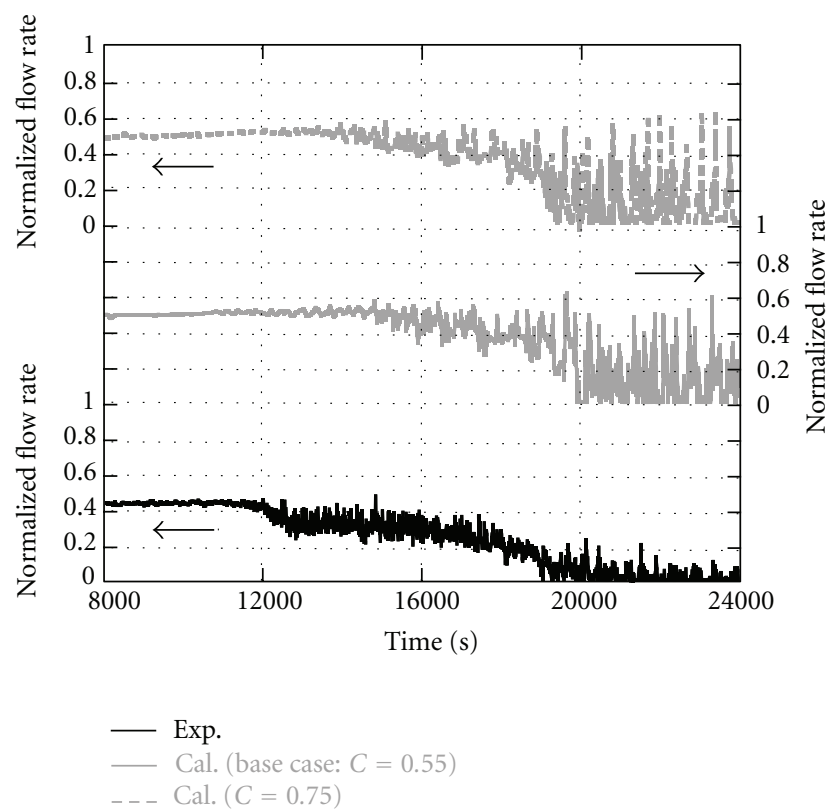

FIGURe 29: RELAP5 results for primary mass flow rate in loop with PZR in cases with different constants $C$ of Wallis CCFL correlation at both all PZR and surge-line junctions.

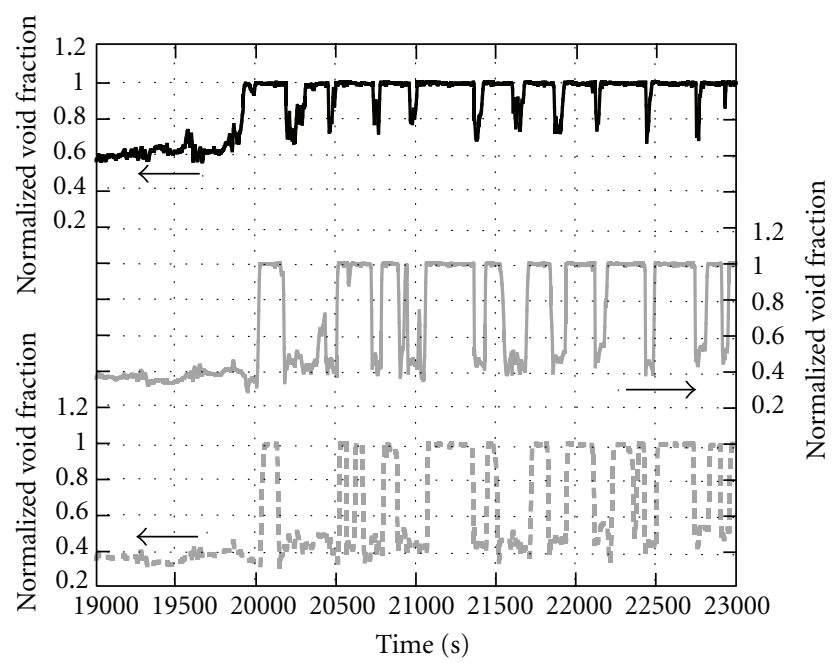

_ Plenum top
_ Plenum middle
_ _ Plenum bottom

FIgURE 30: RELAP5 results for void fraction at top, middle, and bottom of SG inlet plenum in loop with PZR.

Following Wallis correlation [17] was applied to simulate CCFL at both the inlet of U-tubes and inlet plena of SGs:

$$
j_{G}^{* 1 / 2}+m j_{L}^{* 1 / 2}=C,
$$

where $j^{*}$ is the nondimensional volumetric flux. Subscripts $G$ and $L$ denote gas and liquid phases, respectively. Constants of $m$ and $C$ for both the inlet of U-tubes and inlet plena of SGs were set to 1 and 0.75 , respectively, obtained from LSTF experiment on CCFL at the SG U-tube inlet [18]. Influences of the CCFL correlation at the inlet of U-tubes are discussed in Section 6.2.

The PZR was represented by ten vertical nodes to simulate corresponding facility configuration. RELAP5 liquid entrainment model for a horizontal pipe was applied to the PZR surge-line inlet junction connected to the hot leg. Wallis correlation was also applied to simulate CCFL at both all the PZR and the surge-line junctions. Coefficients of $m$ and $C$ of the CCFL correlation were then given as 1 and 0.55 as trial values because of no empirical constants depending on flow channel structure, considering the LSTF test result for the timing when the liquid level was lost in the PZR (Figure 10). Influences of the constants of the CCFL correlation at both all the PZR and the surge-line junctions are discussed in Section 6.2. The core power was decreased down to $75 \%$ when the PCT reached $873 \mathrm{~K}$ as in the experiment. Other initial and boundary conditions were determined according to the LSTF test data.

5.3.2. Comparison of Analysis Results with Experimental Observations. The RELAP5 code predicted well the overall trend of the LSTF experiment for such parameters as primary and secondary pressures due to relatively well predictions of cycle opening of the PORV and the SG RVs as has been compared in Figures 7-21 except Figure 16. The code, however, underpredicted the integrated discharge flow through the PORV (Figure 11) due to earlier change from two-phase flow into single-phase steam discharge because of underprediction of the PZR liquid level (Figure 10) by influences of uncertainties in RELAP5 liquid entrainment model in the hot leg at the inlet of PZR surge-line under high-pressure conditions.

As shown in Figures 14 and 15, large-amplitude level oscillation with some randomness among the SG U-tubes during two-phase natural circulation was qualitatively reproduced by the employed noding with fine-mesh multiple parallel flow channels with a Wallis CCFL correlation at the inlet. Similar to the experiment, the longer tubes tended to have longer intervals between the dumps. However, there were still some differences in both frequency and amplitude of the SG U-tube level oscillation. The SG secondary-side collapsed liquid level and fluid temperature under influences of AFW agreed reasonably well with those in the experiment (Figures 7 and 17).

Two-phase natural circulation was well simulated, though with tendencies that the hot leg liquid level and the primary loop flow rate were overpredicted before about $11500 \mathrm{~s}$ probably due to underprediction of carryover of entrained liquid into the PZR surge-line (Figures 9 and 10). The decrease in the primary loop flow rate with some oscillation was well calculated too, though with a tendency that the oscillation amplitude of the loop flow rate was larger than in the experiment (Figure 9). Larger oscillation in the primary pressure resulted in large level oscillation in the hot 


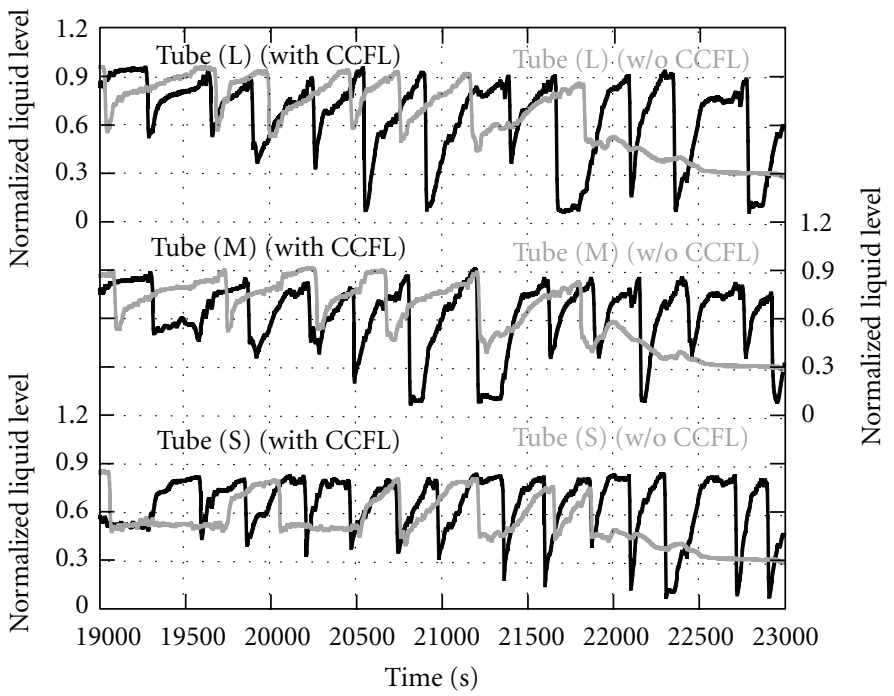

FIGURE 31: RELAP5 results for collapsed liquid levels in upflow side of SG U-tubes in loop with PZR in cases with or without application of Wallis CCFL correlation to inlet of U-tubes.

leg and the SG inlet plenum especially after about $21500 \mathrm{~s}$ (Figures 8, 13, and 18).

The code failed to reproduce the intermittent increase in the cladding surface temperature even before major core uncovery due to failure of large drop in the core liquid level probably because of some discrepancies in the SG U-tube level oscillation. Major core uncovery after the empty of liquid in the upper plenum started somewhat earlier than in the experiment probably due to influences of the differences in the SG U-tube level oscillation (Figures 20 and 21). Then the PCT was about $70 \mathrm{~K}$ higher than in the experiment (Figure 19).

\section{Discussion}

6.1. Comparison with TMLB' Scenario Test Results. The results of this experiment were compared with the TMLB' scenario test results [5] to clarify influences of the different conditions especially for core power and AFW actuation onto the major phenomena. In both experiments, the primaryand SG secondary-side pressures were kept high, respectively, by cycle opening of valves in the PZR and SG secondary-side system as shown in Figure 24. In this experiment, two-phase natural circulation started much earlier than in the TMLB' scenario test due to high core power as shown in Figure 25. Large-amplitude level oscillation also took place in the SG Utubes for a long time while the two-phase natural circulation flow rate gradually decreased with some oscillation because of a continuous primary-to-secondary heat removal by the actuation of AFW. In the TMLB' scenario test, on the contrary, the primary loop flow rate became almost zero when significant drop happened in the SG U-tube liquid levels as shown in Figure 26 after the SG secondary-side became empty of liquid as shown in Figure 27 due to no
AFW. In this experiment, therefore, a temperature excursion happened in the core much later than in the TMLB' scenario test. The influences of AFW were found so significant for the time to start major core uncovery.

6.2. RELAP5 Analysis Results. The CCFL at the PZR bottom may affect the PZR liquid level and the primary loop flow rate. Influences of the constants of the Wallis CCFL correlation at both all the PZR and the surge-line junctions onto the PZR liquid level and the primary loop flow rate were thus investigated as shown in Figures 28 and 29. The analysis with the constant $C$ of the CCFL correlation in (1) to be 0.55 (trial value) was done as the base case, focusing on the timing of empty of liquid in the PZR, and was compared with the case with $C=0.75$ obtained by LSTF experiment for CCFL at the SG U-tube inlet [18] under a common condition of constant $m=1$. The PZR liquid level in the case with the constant $C=0.75$ decreased after about $1400 \mathrm{~s}$ and was lost at about $8770 \mathrm{~s}$ which was much earlier than in the base case. The primary loop flow rate then became almost zero earlier than in the base case due to earlier depletion in the primary coolant inventory. Constants of the CCFL correlation at both all the PZR and the surge-line junctions for the LSTF were thus fixed to be $C=0.55$ and $m=1$.

The calculated results had some differences from the measured data especially in both frequency and amplitude of the SG U-tube level oscillation, though the SG U-tubes were finely modeled as described in Section 5.3.1 with a Wallis CCFL correlation at the inlet of U-tubes. The oscillation amplitude of the primary loop flow rate was also larger than in the experiment. The vertical secondary-side fluid temperature gradient was calculated reasonably well due to good prediction of steam upward flow towards the RVs under a condition that the void fraction in the secondary-side was kept high during the actuation of AFW. 
The calculation revealed that liquid level appears in the SG inlet plenum due to very large void fraction as much as $50 \%$ and the top is filled almost with single-phase steam after around 20000 s, as shown in Figure 30. The calculated results for the SG U-tube liquid levels were compared in the cases with or without application of the Wallis CCFL correlation to the inlet of U-tubes to clarify influences of the CCFL at the tube inlet onto the level oscillation, as shown in Figure 31. Level oscillation amplitude in the tube with the same length in the case without the CCFL became smaller than in the case with the CCFL after around $20000 \mathrm{~s}$. In the case without the CCFL, liquid levels gradually decreased with no oscillation in the tubes in uniform manner after around $22000 \mathrm{~s}$. The CCFL at the inlet of U-tubes has thus affected large-amplitude level oscillation with some randomness among the SG U-tubes for a long time.

When the PWR transients are analyzed with bestestimate computer code, simplified input models are usually employed such that 3382 tubes in each SG are lumped into one equivalent tube to reduce the calculation time. However, a detailed modeling of the SG U-tubes with fine-mesh multiple parallel flow channels would be at least necessary when the complicated two-phase flow phenomena are involved similar to that encountered in the LSTF experiment on the PWR LOFW transient as well as the optimum constants for CCFL correlation to provide better predictions.

\section{Summary}

An LSTF experiment was performed for OECD/NEA ROSA Project, simulating a PWR LOFW transient with specific assumptions of failure of scram to observe high-power natural circulation, and total failure of high pressure injection system. The AFW was provided to well observe the longterm high-power natural circulation. The LSTF core power curve was pre-determined through the RELAP5 code analysis for PWR LOFW transient without scram. The results of the LSTF experiment were compared with the post-test analysis results by the RELAP5 code to clarify the details of major phenomena and remaining subjects in the code predictions. Obtained results are summarized as follows:

(1) Two-phase natural circulation started in very early stage of and continued through the transient where the primary and SG secondary-side pressures were kept almost constant at around the pressures for cycle opening of PORV of PZR and SG RVs. Major core uncovery happened due to core boil-off because of the primary coolant inventory loss through the PORV.

(2) Large-amplitude level oscillation occurred in SG Utubes for a long time in a form of slow fill and dump while the two-phase natural circulation flow rate gradually decreased with some oscillation. The fill and dump behavior depended on the SG U-tube length under influences of vertical fluid temperature distribution along the SG boiler section caused by the AFW coolant injection. Temporary large drop happened in the core liquid level when the water column developed in the SG U-tubes, causing an intermittent increase in the cladding surface temperature at higher elevations even before the major core uncovery due to the core boil-off.

(3) The RELAP5 code predicted well the overall trend of the major phenomena observed in the LSTF experiment including the slow fill and dump behavior in the SG U-tubes. Some discrepancies from the measured data, however, appeared in the oscillative primary loop flow rate and SG U-tube liquid level as well as PZR liquid level. The coefficients of the CCFL correlation suitable for both all the PZR and the surge-line junctions were newly identified.

\section{Acknowledgments}

This paper contains findings gained through the OECD/NEA ROSA Project. It is noted that the value axis of all the data plot of LSTF test and RELAP5 analysis is normalized properly due to the agreement of the OECD/NEA ROSA Project. Authors are also grateful to the Management Board of the ROSA Project for their consent to this publication. Authors would like to thank to Messrs. M. Ogawa and A. Ohwada of Japan Atomic Energy Agency for performing the LSTF experiments under collaboration with members from Nuclear Engineering Co. (NECO) as well as Miss K. Toyoda of IX Knowledge Inc. for manipulating the experimental data.

\section{References}

[1] C. L. Nalezny, "Summary of nuclear regulatory commission's LOFT program experiment," Tech. Rep. NUREG/CR-3214, EGG-2248, 1983.

[2] W. Ambrosini, F. D’Auria, and G. M. Galassi, "Lesson learned from the application to LOBI tests of CATHARE and RELAP5 codes," in Proceedings of the 1st Meeting of the Nuclear Society of Slovenia, Bovec, Slovenia, 1992.

[3] United States Nuclear Regulatory Commission, "Reactor safety study - an assessment of risks in U. S. commercial nuclear power plants," Tech. Rep. WASH-1400 (NUREG075/14), 1975.

[4] The ROSA-V Group, "ROSA-V large scale test facility (LSTF) system description for the third and fourth simulated fuel assemblies," JAERI-Tech 2003-037, Japan Atomic Energy Research Institute, Ibaraki, Japan, 2003.

[5] Y. Kukita, Y. Anoda, and K. Tasaka, "Summary of ROSA-IV LSTF first-phase test program-integral simulation of PWR small-break LOCAs and transients," Nuclear Engineering and Design, vol. 131, no. 1, pp. 101-111, 1991.

[6] K. E. Carlson, "RELAP5/MOD3 code manual (draft)," Tech. Rep. NUREG/CR-5535, EGG-2596, 1990.

[7] T. Takeda, H. Asaka, and H. Nakamura, "Analysis of the OECD/NEA ROSA project experiment simulating a PWR small break LOCA with high-power natural circulation," Annals of Nuclear Energy, vol. 36, no. 3, pp. 386-392, 2009.

[8] H. Nakamura, T. Watanabe, T. Takeda et al., "RELAP5/MOD3 code verification through PWR pressure vessel small break loca tests in OECD/NEA rosa project," in Proceedings of the 
16th International Conference on Nuclear Engineering (ICONE16 '08), pp. 659-668, Orlando, Fla, USA, May 2008.

[9] N. Zuber, "Problems in modeling small break LOCA," USNRC Report NUREG-0724, 1980.

[10] H. Kumamaru and K. Tasaka, "Recalculation of simulated post-scram core power decay curve for use in ROSA-IV/LSTF experiments on PWR small-break LOCAs and transients," Tech. Rep. JAERI-M 90-142, Japan Atomic Energy Research Institute, Ibaraki, Japan, 1990.

[11] ROSA Project Team, "Final integration report of OECD/NEA ROSA project," JAEA-Research 2010-9002, Japan Atomic Energy Agency, Ibaraki, Japan, 2010.

[12] Y. Kukita, H. Nakamura, K. Tasaka, and C. Chauliac, "Nonuniform steam generator U-tube flow distribution during natural circulation tests in ROSA-IV large scale test facility," Nuclear Science and Engineering, vol. 99, no. 4, pp. 289-298, 1988.

[13] H. Asaka, Y. Kukita, T. Yonomoto, Y. Koizumi, and K. Tasaka, "Results of $0.5 \%$ cold-leg small-break LOCA experiments at ROSA-IV/LSTF: effect of break orientation," Experimental Thermal and Fluid Science, vol. 3, no. 6, pp. 588-596, 1990.

[14] K. H. Ardron and R. A. Furness, "A study of the critical flow models used in reactor blowdown analysis," Nuclear Engineering and Design, vol. 39, no. 2-3, pp. 257-266, 1976.

[15] D. W. Sallet, "Thermal hydraulics of valves for nuclear applications," Nuclear Science and Engineering, vol. 88, no. 3, pp. 220-244, 1984.

[16] Susyadi and T. Yonomoto, "Analysis on non uniform flow in steam generator during steady state natural circulation cooling," JAERI-Research 2005-011, Japan Atomic Energy Research Institute, Ibaraki, Japan, 2005.

[17] G. B. Wallis, One-Dimensional Two-Phase Flow, McGraw-Hill Book, New York, NY, USA, 1969.

[18] T. Yonomoto, Y. Anoda, Y. Kukita, and Y. Peng, "CCFL characteristics of PWR steam generator U-tubes," in Proceedings of the ANS International Topical Meeting on Safety of Thermal Reactors, American Nuclear Society, Portland, Ore, USA, 1991. 

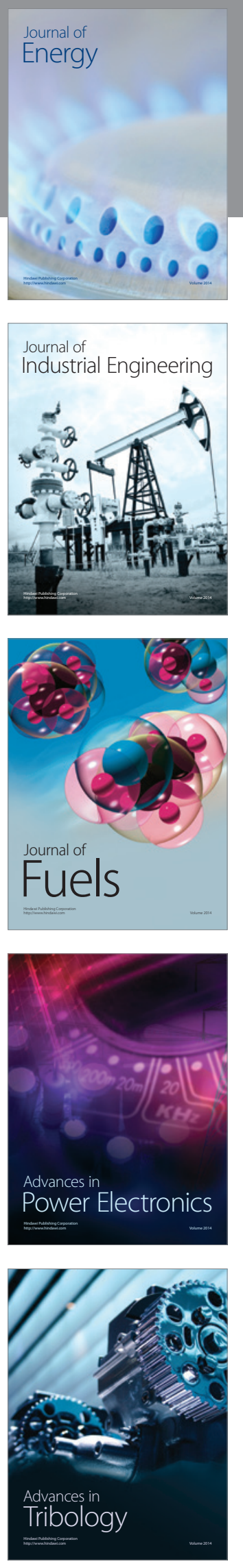
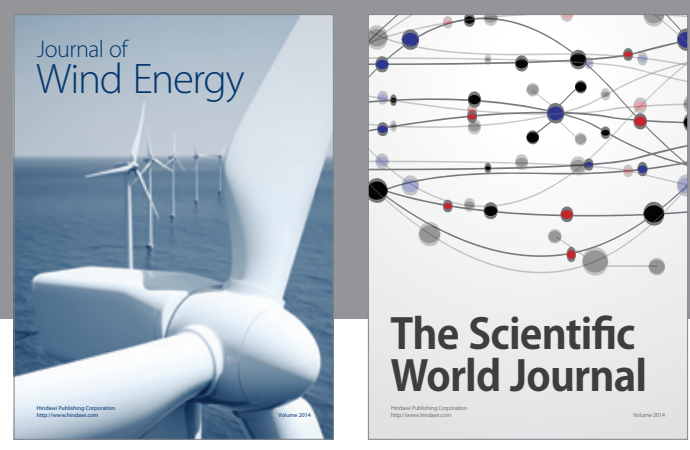

The Scientific World Journal

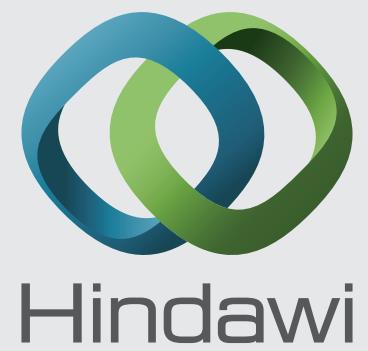

Submit your manuscripts at http://www.hindawi.com
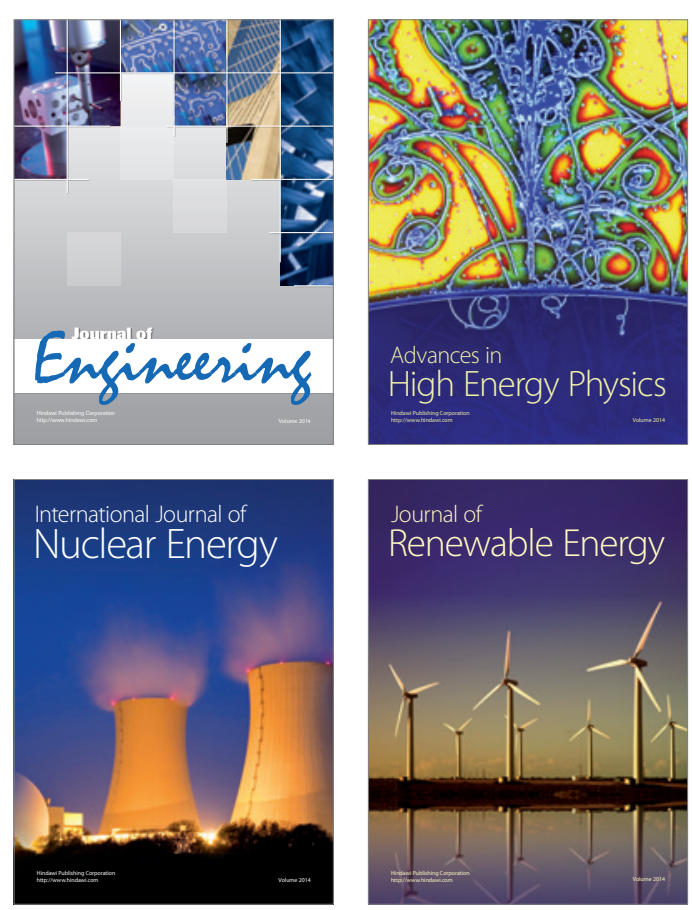

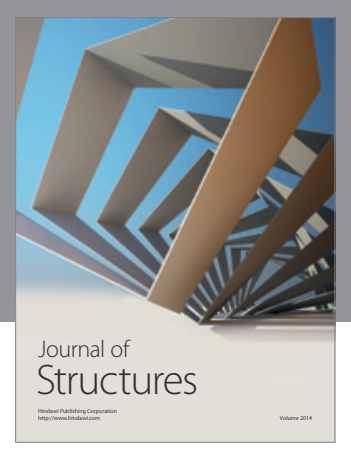

Rotating
Mechinery
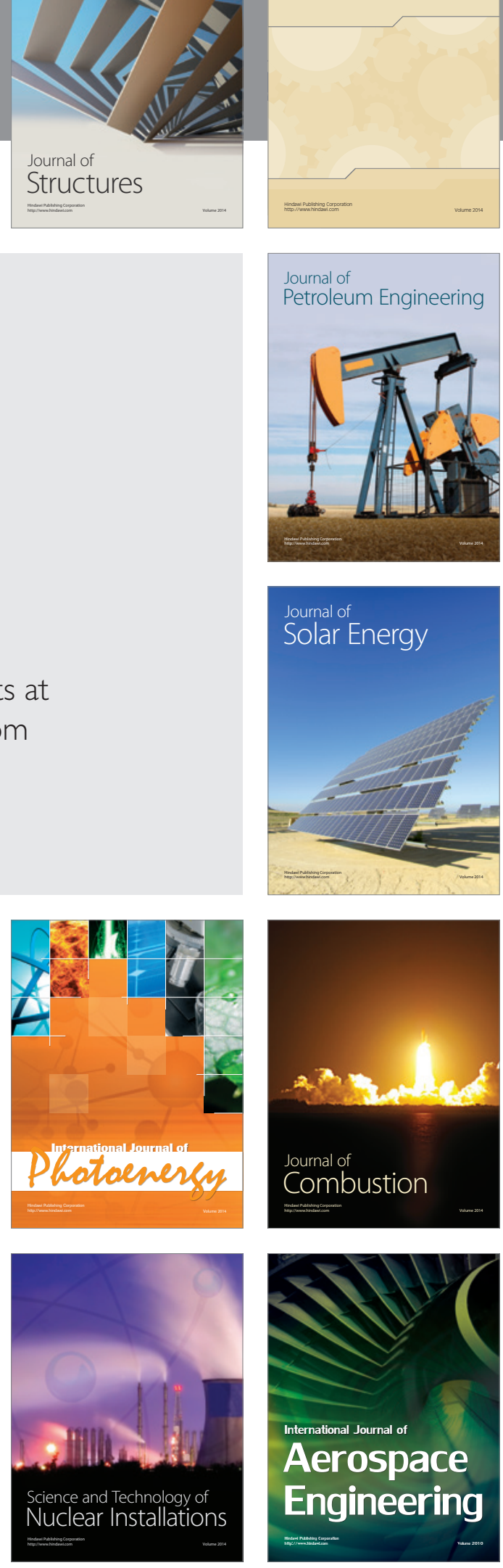